Article

\title{
Comprehensive Chemical Profiling and Multidirectional Biological Investigation of Two Wild Anthemis Species (Anthemis tinctoria var. Pallida and A. cretica subsp. tenuiloba): Focus on Neuroprotective Effects
}

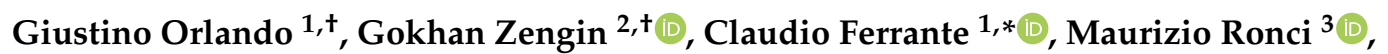 \\ Lucia Recinella ${ }^{1}$, Ismail Senkardes ${ }^{4}$, Reneta Gevrenova ${ }^{5}$, Dimitrina Zheleva-Dimitrova ${ }^{5}$, \\ Annalisa Chiavaroli ${ }^{1}$, Sheila Leone ${ }^{1}$, Simonetta Di Simone ${ }^{1}$, Luigi Brunetti ${ }^{1}$, \\ Carene Marie Nancy Picot-Allain ${ }^{6}$, Mohamad Fawzi Mahomoodally ${ }^{6} \mathbb{D}$, \\ Kouadio Ibrahime Sinan ${ }^{2}$ and Luigi Menghini ${ }^{1}$ (D) \\ 1 Department of Pharmacy, University "G. d'Annunzio" of Chieti-Pescara, 66100 Chieti, Italy \\ 2 Department of Biology, Faculty of Science, Selcuk University, Konya 42130, Turkey \\ 3 Department of Medical, Oral and Biotechnological Sciences, University “G. d'Annunzio" of Chieti-Pescara, \\ 66100 Chieti, Italy \\ 4 Department of Pharmaceutical Botany, Faculty of Pharmacy, Marmara University, Istanbul 34668, Turkey \\ 5 Department of Pharmacognosy, Faculty of Pharmacy, Medical University of Sofia, 1431 Sofia, Bulgaria \\ 6 Department of Health Sciences, Faculty of Science, University of Mauritius, Réduit 80837, Mauritius \\ * Correspondence: claudio.ferrante@unich.it; Tel.: +39-0871-355-4755 \\ + These authors contributed equally to this work.
}

Academic Editors: Raffaele Capasso and Lorenzo Di Cesare Mannelli Received: 1 July 2019; Accepted: 14 July 2019; Published: 16 July 2019

\begin{abstract}
Ethyl acetate (EA), methanol (MeOH), and aqueous extracts of aerial parts of Anthemis tinctoria var. pallida (ATP) and A. cretica subsp. tenuiloba (ACT) were investigated for their phenol and flavonoid content, antioxidant, and key enzyme inhibitory potentials. All extracts displayed antiradical effects, with $\mathrm{MeOH}$ and aqueous extracts being a superior source of antioxidants. On the other hand, EA and $\mathrm{MeOH}$ extracts were potent against $\mathrm{AChE}$ and BChE. Enzyme inhibitory effects against tyrosinase and $\alpha$-glucosidase were observed, as well. We also studied Anthemis extracts in an ex vivo experimental neurotoxicity paradigm. We assayed extract influence on oxidative stress and neurotransmission biomarkers, including lactate dehydrogenase (LDH) and serotonin (5-HT), in isolated rat cortex challenged with $\mathrm{K}^{+} 60 \mathrm{mM}$ Krebs-Ringer buffer (excitotoxicity stimulus). An untargeted proteomic analysis was finally performed in order to explore the putative mechanism in the brain. The pharmacological study highlighted the capability of ACT water extract to blunt $\mathrm{K}^{+} 60 \mathrm{mM}$ increase in LDH level and 5-HT turnover, and restore physiological activity of specific proteins involved in neuron morphology and neurotransmission, including NEFMs, VAMP-2, and $\mathrm{PKC} \gamma$, thus further supporting the neuroprotective role of ACT water extract.
\end{abstract}

Keywords: Anthemis; oxidative stress; neurotransmission; proteomic; phytomedicine

\section{Introduction}

Anthemis L is the second largest genus in Asteraceae family including more than 210 species, which are distributed in western Eurasia, Mediterranean and a small part of eastern Africa. According to the Flora of Turkey, the Anthemideae are divided into three subgenera (Anthemis, Maruta and Cota) and the subgenus Anthemis includes four sections; Hiorthia, Anthemis, Maruta, and Chia [1-3]. In Turkey, 
the genus is represented by 81 taxa belonging to 51 species, 29 (54\%) of which are endemic. Species belonging to Anthemis genus are commonly referred to as "Papatya", in Turkey $[1,4,5]$.

The species belonging to Anthemis genus are known to possess various biological properties and have found broad use in pharmaceutics, cosmetics, and food chemistry. The flowers of Anthemis species are well-documented for their use as antiseptic and healing herbs, with flavonoids, and essential oils being the main active components [2,6]. Extracts, tinctures, salves, and tisanes are extensively used as antispasmodic, anti-inflammatory, antibacterial and sedative agents, in Europe [5]. Extracts are also used to clean wounds and ulcers, and as therapy for irradiated skin injuries, cystitis and dental afflictions [2]. The antimicrobial activity of essential oils of several Anthemis species have been previously reported [7-10]. Moreover, Anthemis species are widely used to treat intestinal disorders, kidney stones, and hemorrhoids in traditional medicine. The plant is also used as antispasmodic medications and to stimulate menstrual flow. It is documented that the seed oil has been used in the treatment of earaches and deafness [11-13].

Anthemis genus is mainly characterized by the presence of sesquiterpene lactones, flavonoids and essential oils. Sesquiterpene lactones belonging to germacranolides, eudesmanolides, and guaianolides have been gained attention because of their chemo-ecological functions, biological activities and taxonomic significance. They are the major classes of secondary metabolites in Anthemis genus [2,3,14]. The essential oil compositions of several Anthemis species has also been investigated $[2,12,15,16]$.

A. tinctoria var. pallida (ATP) is a rounded perennial plant measuring between 20 to $45 \mathrm{~cm}$. The flowers are white or cream [17]. ATP, commonly known as yellow chamomile, produces a yellow dye used in food industry for production of diary and butchery products. Decoction of ATP flower is traditionally taken to treat shortness of breath, bronchitis, stomachache, anxiety, and to strengthen hair [18]. Aerial part of Anthemis species has been reported to exhibit antimicrobial property [17]. However, there is no record of the use of A. cretica subsp. tenuiloba (ACT) by folk populations, in Turkey.

To the best of our knowledge, there are no reports in literature investigating chemical profile and biological activities of ATP and ACT. Thus, we aimed to determine the chemical characterization and biological effects of these two Anthemis species. Phytochemical profiles of ethyl acetate (EA), methanol $(\mathrm{MeOH})$ and aqueous extracts were performed by ultra-high-performance liquid chromatography coupled with electrospray ionization high resolution mass spectrometry (UHPLC-ESI/HRMS). The samples were assayed for evaluating antioxidant and enzyme inhibitory potential, as well.

Finally, considering both the traditional antianxiety effect of $A$. tinctoria, the relationships between anxiety and brain oxidative/inflammatory stress [19], alongside with the well-established multi-target protective effects exerted by flavonoid fraction, in the brain [20], we studied the putative protective role of Anthemis extracts in isolated rat cortex challenged with a neurotoxicity stimulus $\left(\mathrm{K}^{+} 60 \mathrm{mM}\right)$. The influence of extract supplementation on the levels of specific biomarkers of oxidative stress and neurotransmission, including lactate dehydrogenase (LDH) and serotonin (5-HT), was investigated using validated analytical methods. An untargeted proteomic profile was also performed on rat cortex homogenate, in order to explore the putative mechanism of action of Anthemis extracts. It is expected that results presented in this study will support the protective effects of the studied Anthemis extracts as potential pharmacological agents.

\section{Results and Discussion}

\subsection{Total Phenolic and Flavonoid Contents}

Phenolic compounds are of increasing interest mainly due to their diverse chemical structure and wide biological activity valuable in the prevention of some chronic or degenerative diseases. To this end, the evaluation of the phytochemical profile of plant extracts is important. In the present study, the total phenol and flavonoid contents of $\mathrm{EA}, \mathrm{MeOH}$, and aqueous extracts of ATP and ACT were illustrated in Table 1. The phenolic content of ATP and ACT ranged from 26.46 to $100.09 \mathrm{mg}$ GAE/g and 21.31 to $47.61 \mathrm{mg} \mathrm{GAE} / \mathrm{g}$, respectively. Highest phenolic content was observed in the $\mathrm{MeOH}$ extract of 
ATP, followed by its aqueous extract. Whilst for ACT, MeOH extract contained the highest amount of phenols, followed by EA extract. Regarding the total flavonoid content, the results showed that EA (ATP: 45.82 an ACT: $46.26 \mathrm{mg}$ RE/g) and MeOH (ATP: 48.54 and ACT: $45.08 \mathrm{mg}$ RE/g) extracts of both species were rich in flavonoids.

Table 1. Total phenol and flavonoid content of Anthemis extracts*.

\begin{tabular}{cccc}
\hline Plant Names & Solvents & $\begin{array}{c}\text { Total Phenol Content } \\
\text { (mg GAE/g) }\end{array}$ & $\begin{array}{c}\text { Total Flavonoid } \\
\text { Content (mg RE/g) }\end{array}$ \\
\hline \multirow{3}{*}{ A. tinctoria var. pallida } & EA & $26.46 \pm 1.11^{\mathrm{d}}$ & $45.82 \pm 0.40^{\mathrm{b}}$ \\
& $\mathrm{MeOH}$ & $100.09 \pm 2.83^{\mathrm{a}}$ & $48.54 \pm 0.57^{\mathrm{a}}$ \\
& Aqueous & $86.74 \pm 1.80^{\mathrm{b}}$ & $23.10 \pm 0.13^{\mathrm{d}}$ \\
\hline \multirow{3}{*}{ A. cretica subsp. tenuiloba } & $\mathrm{EA}$ & $21.31 \pm 1.58^{\mathrm{e}}$ & $46.26 \pm 0.25^{\mathrm{b}}$ \\
& $\mathrm{MeOH}$ & $46.73 \pm 0.80^{\mathrm{c}}$ & $45.08 \pm 0.26^{\mathrm{c}}$ \\
& Aqueous & $47.61 \pm 1.89^{\mathrm{c}}$ & $21.17 \pm 0.24^{\mathrm{e}}$ \\
\hline
\end{tabular}

* Values expressed are means \pm S.D. of three parallel measurements. GAE: Gallic acid equivalent; RE: Rutin equivalent. Different letters indicate significant differences in the extracts $(p<0.05)$.

\subsection{LC-MS Results}

In the present study, 70 compounds were tentatively identified by UHPLC-ESI/MS in both ACT and ATP extracts. The negative ion mode was used for analysis of acylquinic acids and flavonoids, while positive ion mode was used for sesquiterpenes determination (Table 2).

\subsubsection{Acylquinic Acids}

Twenty nine acylquinic acids were identified in tested Anthemis extracts (Table 2). The acylquinic acids elucidation was based on the hierarchical key developed by Clifford and colleagues [21,22]. Peaks 4, 5, 6, and 7 were identified as 3-O-, 1-O-, 5-O- and 4-O-caffeoylquinic acids ([M - H $]^{-}$at $\mathrm{m} / \mathrm{z} 353.088$ ), respectively, according to the relative abundance of the characteristic fragment ions at $\mathrm{m} / \mathrm{z} 191.055$ [quinic acid $-\mathrm{H}]^{-}, 179.034$ [caffeic acid $\left.-\mathrm{H}\right]^{-}, 173.045$ [quinic acid $-\mathrm{H}-\mathrm{H}_{2} \mathrm{O}^{-}$, and 135.044 [caffeic acid $\left.-\mathrm{H}-\mathrm{CO}_{2}\right]^{-}[21,22]$. Compounds 4 and $\mathbf{6}$ were identified by comparison with neochlorogenic and chlorogenic acid, respectively. In the same manner, peaks $\mathbf{1}, \mathbf{2}$, and $\mathbf{3}$ were assigned as 3-O-, 5-O-, and 1-O-p-coumaroylquinic acids ([M - H $]^{-}$at $m / z$ 337.093), while peaks 8, 9, 10, and $\mathbf{1 1}\left([\mathrm{M}-\mathrm{H}]^{-}\right.$at $m / z$ 367.103) were assigned as 3-O-, 1-O- 5-O-, and 4-O-feruloylquinic acid (Table 4). With respect to the diacylquinic acids, peaks 12-15 were related to 3,4-O-, 1,5-O, 3,5-O, and 4,5-O-dicaffeoylquinic acids $\left([\mathrm{M}-\mathrm{H}]^{-}\right.$at $m / z$ 515.120); $\mathbf{1 2}$ and $\mathbf{1 3}$ were identified by comparison with standards. The presence of $\mathbf{1 4}$ was evidenced by the relative abundance of the ions at $m / z 191.055,179.034$, and 135.043 [21-23], while the ion at 173.044 was prominent for 15. Compounds 16-24, $[\mathrm{M}-\mathrm{H}]^{-}$at $m / z 529.136$ were tentatively identified as caffeoylferuloylquinic acids [21,22]. Among the tricaffeoylquinic acids, peaks 25-29 were related to $\left([\mathrm{M}-\mathrm{H}]^{-}\right.$at $m / z$ 677.152). Compounds 26, 28, and 29 yielded indicative fragment ions at 173.045 deduced 4-substituted CQA [22]. According to the presence of weak signal at $\mathrm{m} / \mathrm{z} 203.034$, the relative intensity of the fragment ion at $m / z$ 335.078, and lipophilicity, peaks 26, 28, and 29 were tentatively assigned as 1,3,4-O-, 1,4,5-O-, and 3,4,5-O-tricaffeoylquinic acid, while 25 and 27 were related to 3,4,5-O-tricaffeoylquinic acid and its isomer.

\subsubsection{Flavonoids}

Based on literature and comparison with standards, 15 flavonoid aglycones $\mathbf{3 0 - 4 4}$ (most of them methoxylated), twelve glycosides, and one caffeoyl-O-flavonoid were identified in the studied extracts (Table 4). Regarding 41-43 ([M - H $]^{-}$at $\left.m / z 345.061\right)$, the fragment ion at $m / z 287.020$, due to consecutive loss of $2 \mathrm{CH}_{3}{ }^{-}$and $\mathrm{CO}$ is more intense in the product-ion spectra of $\mathbf{4 3}$ than $\mathbf{4 1}$ and $\mathbf{4 2}$. Probably methoxylation of $\mathbf{4 3}$ in both A- and C-rings provides very stable fragments due to concurrent methyl loss [24]. Fragment ion at $m / z 121.028\left({ }^{1,2} \mathrm{~B}\right)$ (for 41 and 42$)$ were attributed to the Retro-Diels Alder 
(RDA) cleavages of the flavonoid skeleton specific for $3^{\prime}, 4^{\prime}$-dihydroxy flavonols [25]. Thus, according to literature, 41-43 were tentatively identified as eupatolitin, spinatoside, and spinacetin, respectively.

The fragmentation fingerprints of $\mathbf{5 2}$ and $\mathbf{5 6}$ were associated with isorhamnetin derivatives, witnessed by the abundant fragment ion at $\mathrm{m} / \mathrm{z} 315.051$ supported by the ions at $\mathrm{m} / \mathrm{z} 300.027$ and 133.028 [24]. Fragmentation patterns and monoisotopic profiles of 52 was in good agreement with those of caffeoyl-O-isorhamnetin. The fragmentation of [M- $\mathrm{H}]^{-}$at $\mathrm{m} / \mathrm{z} 609.1472$ (56) yielded abundant ion at $\mathrm{m} / \mathrm{z} 315.0517\left([\mathrm{M}-\mathrm{H}-294.095]^{-}\right.$indicating the loss of hexose and pentose moieties.

\subsubsection{Sesquiterpenes}

Thirteen sesquiterpene lactones including one eudesmanolide, three germacranolides, and nine guaianolides, were tentatively identified in both ACT and ATP extracts. Concerning compound 58 ([M $+\mathrm{H}^{+}$at $\left.\mathrm{m} / \mathrm{z} 229.122\right)$, its fragmentation pattern involved losses of $18 \mathrm{Da}\left(\mathrm{H}_{2} \mathrm{O}\right), 28 \mathrm{Da}(\mathrm{CO})$ and $46 \mathrm{Da}$ $\left(\mathrm{CO}_{2} \mathrm{H}\right)$ suggesting chamazulene carboxylic acid, a degradation product of proazulenic sesquiterpene lactones, e.g., matricarin [25]. Similar fragmentation patterns were observed in spectra of $\mathbf{5 9}$ and $\mathbf{6 0}$. In addition, a loss of $44 \mathrm{Da}\left(\mathrm{CO}_{2}\right)$ and fragment ions at $m / z 185.095\left([\mathrm{M}+\mathrm{H}-16-44]^{+}\right.$and $95.049\left(\mathrm{C}_{6} \mathrm{H}_{7} \mathrm{O}\right)$ due to the overall fracture of lactone ring, suggested dehydroleucodin or isodehydroleucodin [26]. Accordingly, 61 was assigned as leucodin $\left([\mathrm{M}+\mathrm{H}]^{+}\right.$at $\left.\mathrm{m} / \mathrm{z} 247.132\right)$, where $\mathrm{C}-13$ was saturated in a methyl group. 68 and 69 were tentatively identified as matricarin and its isomer, due to the concomitant loss of $\left(\mathrm{CO}_{2}+\mathrm{H}_{2} \mathrm{O}\right)$ at $\mathrm{m} / \mathrm{z} 245.117$ from the additional acetyl group [3]. Three isobaric sesquiterpene lactones 63-65 shared the same $[\mathrm{M}+\mathrm{H}]^{+}$at $\mathrm{m} / z 263.127$ (exact mass). Peaks 63-65 demonstrated difference of $15.995 \mathrm{Da}$, in comparison to $\mathbf{6 1}$, suggesting the presence of an additional hydroxyl group. Thus 63-65 were tentatively assigned to hydroxyleucodin and its isomers [27]. In the same manner, peaks 62 , isobaric pair $66 / 67$, and 70 were ascribed as parthenolide, stizolin, and ludalbine, respectively, previously identified in Anthemis species [3].

\subsection{Antioxidant Activity}

Oxidative stress-related diseases often arise as a result of the imbalance between the production of free radicals and reactive oxygen/nitrogen species, and antioxidant defences. These diseases can be managed/prevented using natural antioxidants that represent promising therapeutic candidates [29]. Different antioxidant assays are needed to obtain certain information regarding antioxidant profile of herbal extracts. From this point, the antioxidant capacity of different extracts of ATP and ACT were evaluated using multiple assays based on different mechanisms and the results were presented in Table 3. 
Table 2. Peak assessment of compounds in Anthemis extracts.

\begin{tabular}{|c|c|c|c|c|c|c|c|c|}
\hline Peak № & $\begin{array}{l}\text { Accurate Mass } \\
{[\mathrm{M}-\mathrm{H}]^{-} \mathrm{m} / \mathrm{z}}\end{array}$ & $\begin{array}{l}\text { Molecular } \\
\text { Formula }\end{array}$ & MS/MS Data $m / z$ & $t_{R} \min$ & $\begin{array}{l}\text { Exact Mass } \\
{[\mathbf{M}-\mathbf{H}]^{-} m / z}\end{array}$ & Delta ppm & Tentative Structure & Ref. \\
\hline \multicolumn{9}{|c|}{ Acylquinnic acids } \\
\hline \multicolumn{9}{|c|}{ Monoacylquinic acids } \\
\hline 1 & 337.0946 & $\mathrm{C}_{16} \mathrm{H}_{17} \mathrm{O}_{8}$ & $\begin{array}{c}337.0946(3.1), 191.0557(7.7), 173.0453(2.5), 163.0390 \\
(100), 119.0488(22.8)\end{array}$ & 3.05 & 337.0929 & 5.071 & $\begin{array}{l}\text { 3-p-coumaroyl-quinic } \\
\text { acid }^{1,2,6}\end{array}$ & [19] \\
\hline 2 & 337.0932 & $\mathrm{C}_{16} \mathrm{H}_{17} \mathrm{O}_{8}$ & $\begin{array}{c}337.0932 \text { (7.2), } 191.0554 \text { (100), } 173.0445 \text { (6.7), } 163.0390 \\
(5.8), 119.0489(5.5), 93.0329(17.6)\end{array}$ & 4.07 & 337.0929 & 0.829 & $\begin{array}{l}5 \text { - } p \text {-coumaroyl-quinic } \\
\text { acid } 1,2,3,5,6\end{array}$ & [19] \\
\hline 3 & 337.0930 & $\mathrm{C}_{16} \mathrm{H}_{17} \mathrm{O}_{8}$ & $\begin{array}{c}337.0930(5.9), 191.0554(100), 173.0446(1.9), 163.0391 \\
(2.4), 127.0391(1.5), 119.0487(1.4), 111.0439(1.3) \\
93.0331(5.3), 85.0280(7.1)\end{array}$ & 4.74 & 337.0929 & 0.473 & $\begin{array}{l}\text { 1-p-coumaroyl-quinic } \\
\text { acid } \\
1,2,5,6\end{array}$ & [19] \\
\hline 4 & 353.0879 & $\mathrm{C}_{16} \mathrm{H}_{17} \mathrm{O}_{9}$ & $\begin{array}{c}353.0879(32.4), 191.0553(100), 179.0341(60.7) \\
173.0443(4.1), 161.0233(3.9), 135.0439(50.7), 111.0438 \\
(0.9), 93.0333(4.5), 85.0279(8.7)\end{array}$ & 2.47 & 353.0867 & 0.325 & $\begin{array}{c}\text { neochlorogenic } \\
\text { (3-caffeoylquinic) acid } \\
1,2,3,4,6\end{array}$ & $*$ \\
\hline 5 & 353.0879 & $\mathrm{C}_{16} \mathrm{H}_{17} \mathrm{O}_{9}$ & $\begin{array}{c}353.0879(29.9), 191.0554(47.7), 179.0341 \text { (70.1), } \\
173.0447(100), 135.0439(57.7), 111.0436(3.3), 93.331 \\
(22.6), 85.0280(8.6)\end{array}$ & 2.48 & 353.0867 & 0.240 & 1-caffeoylquinic acid & [19] \\
\hline 6 & 353.0880 & $\mathrm{C}_{16} \mathrm{H}_{17} \mathrm{O}_{9}$ & $\begin{array}{c}353.0880(3.8), 191.0554(100), 179.0338(1.4), 173.0446 \\
(0.9), 161.0234(2.1), 135.0441(1.1), 111.0435(1.2) \\
93.0331(2.7), 85.0279(0.4)\end{array}$ & 3.30 & 353.0867 & 0.495 & $\begin{array}{c}\text { chlorogenic } \\
\text { (5-caffeoylquinic) acid } \\
1,2,3,4,5,6\end{array}$ & $*$ \\
\hline 7 & 353.0880 & $\mathrm{C}_{16} \mathrm{H}_{17} \mathrm{O}_{9}$ & $\begin{array}{c}353.0880(28.1), 203.6057(0.1), 191.0554(100), 179.0341 \\
(58.3), 173.0446(4.6), 161.0233(3.9), 135.0438(51.8) \\
111.0437(2.2), 93.0330(4.82), 85.0279(10.2)\end{array}$ & 5.92 & 353.0867 & 0.665 & $\begin{array}{l}\text { 4-caffeoylquinic acid } \\
1,2,3,4,6\end{array}$ & [19] \\
\hline 8 & 367.1043 & $\mathrm{C}_{17} \mathrm{H}_{19} \mathrm{O}_{9}$ & $\begin{array}{c}367.1043(12.5), 193.0500(100), 173.0453(3.9), 149.0598 \\
(2.9), 134.0361(65.7), 127.0395(1.0), 111.0439(1.4) \\
93.0331(2.9)\end{array}$ & 3.54 & 367.1034 & 2.410 & $\begin{array}{l}\text { 3-feruloylquinic acid } \\
1,2,5,6\end{array}$ & [19] \\
\hline 9 & 367.1039 & $\mathrm{C}_{17} \mathrm{H}_{19} \mathrm{O}_{9}$ & $\begin{array}{c}367.1039(49.3), 161.0234 \text { (100), } 127.0390 \text { (1.7), } 85.0281 \\
\text { (13.4) }\end{array}$ & 3.97 & 367.1034 & 1.238 & 1-feruloylquinic acid & [19] \\
\hline 10 & 367.1035 & $\mathrm{C}_{17} \mathrm{H}_{19} \mathrm{O}_{9}$ & $\begin{array}{c}367.1035(14.1), 193.0499(6.7), 191.0555(100), 173.0447 \\
(18.2), 134.0447(10.9), 111.0437(3.9), 93.0331(26.2) \\
85.0280(5.3)\end{array}$ & 4.52 & 367.1034 & -0.015 & $\begin{array}{l}\text { 5-feruloylquinic acid } \\
\qquad 1,2,3,4,5,6\end{array}$ & \\
\hline 11 & 367.1030 & $\mathrm{C}_{17} \mathrm{H}_{19} \mathrm{O}_{9}$ & $\begin{array}{c}367.1030(11.8), 193.0499(17.1), 173.0446(100), \\
111.0435(3.1), 93.0331(24.0)\end{array}$ & 4.78 & 367.1034 & -1.322 & 4-feruloylquinic acid & [19] \\
\hline
\end{tabular}


Table 2. Cont.

\begin{tabular}{|c|c|c|c|c|c|c|c|c|}
\hline Peak № & $\begin{array}{c}\text { Accurate Mass } \\
{[\mathbf{M}-\mathbf{H}]^{-} m / z}\end{array}$ & $\begin{array}{l}\text { Molecular } \\
\text { Formula }\end{array}$ & MS/MS Data $m / z$ & $t_{R} \min$ & $\begin{array}{c}\text { Exact Mass } \\
{[\mathbf{M}-\mathbf{H}]^{-} m / z}\end{array}$ & Delta ppm & Tentative Structure & Ref. \\
\hline \multicolumn{9}{|c|}{ Diacylquinic acids } \\
\hline 12 & 515.1196 & $\mathrm{C}_{25} \mathrm{H}_{23} \mathrm{O}_{12}$ & $\begin{array}{c}515.1196(100), 353.0880(18.5), 335.0788(6.4), 203.0331 \\
(1.3), 191.0554(29.5), 179.0341(58.1), 173.0446(65.7) \\
161.0235(19.8), 135.0439(57.5), 127.0389(3.6) \\
111.0436(4.7), 93.0330(18.7), 85.0277(4.3)\end{array}$ & 5.79 & 515.1184 & 0.137 & $\begin{array}{l}\text { 3,4-dicaffeoylquinic acid } \\
\qquad, 2,3,4,5,6\end{array}$ & * \\
\hline 13 & 515.1199 & $\mathrm{C}_{25} \mathrm{H}_{23} \mathrm{O}_{12}$ & $\begin{array}{c}515.1199(21.8), 353.0881(83.1), 335.0782(2.6) \\
191.0554(100), 179.0341(48.6), 173.0445(10.6) \\
161.0233(12.4), 135.0439(57.5), 127.0387(2.8), 93.0332 \\
(6.4), 85.0280(9.0)\end{array}$ & 5.96 & 515.1184 & & $\begin{array}{l}\text { 1,5-dicaffeoylquinic acid } \\
1,2,3,4,5,6\end{array}$ & $*$ \\
\hline 14 & 515.1204 & $\mathrm{C}_{25} \mathrm{H}_{23} \mathrm{O}_{12}$ & $\begin{array}{c}515.1204(22.6), 353.0880(87.4), 191.0555(100), \\
179.0342(52.0), 173.0455(9.8), 161.0235(8.9), 135.0438 \\
(51.7), 93.0333(2.8), 85.0281(8.6),\end{array}$ & 6.14 & 515.1184 & 1.787 & 3,5-dicaffeoylquinic acid 3,4 & [28] \\
\hline 15 & 515.1199 & $\mathrm{C}_{25} \mathrm{H}_{23} \mathrm{O}_{12}$ & $\begin{array}{c}515.1199(75.6), 353.0880(60.6), 203.0343(3.1) \\
191.0555(34.7), 179.0342(72.3), 173.0447(100) \\
135.0440(48.1), 127.0384(1.2), 111.0439(3.7), 93.0330 \\
(17.2), 85.0280(4.2)\end{array}$ & 6.34 & 515.1184 & 0.720 & $\begin{array}{l}\text { 4,5-dicaffeoylquinic acid } \\
\qquad, 2,3,4,5,6\end{array}$ & [28] \\
\hline 16 & 529.1356 & $\mathrm{C}_{26} \mathrm{H}_{25} \mathrm{O}_{12}$ & $\begin{array}{c}529.1356(100), 367.1037(8.3), 353.0889(7.4), 349.0935 \\
(5.2), 335.0774(11.8), 193.0499(60.5), 191.0555(9.2), \\
179.0342(42.1), 173.0446(48.1), 161.0235(24.6) \\
149.0596(1.1), 134.0361(56.0), 111.0437(8.9), 93.0331 \\
(14.7), 85.0276(2.7)\end{array}$ & 6.00 & 529.1351 & 0.889 & $\begin{array}{l}\text { 3-feruloyl-5-caffeoylquinic } \\
\text { acid } 1,2,4,5,6\end{array}$ & [28] \\
\hline 17 & 529.1357 & $\mathrm{C}_{26} \mathrm{H}_{25} \mathrm{O}_{12}$ & $\begin{array}{c}529.1357(55.4), 367.1038(25.6), 193.0499(2.6) \\
179.0342(3.0), 173.0449(1.9), 161.0234(100), 135.0441 \\
(12.7), 134.0367(4.3), 127.0380(0.5), 93.0331(1.1) \\
85.0279(3.2)\end{array}$ & 6.92 & 529.1351 & 1.003 & $\begin{array}{l}\text { 1-feruloyl-5-caffeoylquinic } \\
\text { acid } 1,2,3,5\end{array}$ & [28] \\
\hline 18 & 529.1354 & $\mathrm{C}_{26} \mathrm{H}_{25} \mathrm{O}_{12}$ & $\begin{array}{c}529.1354 \text { (38.3), } 367.1040 \text { (39.6), } 353.0883(44.99), \\
193.0506 \text { (15.6), } 191.0555 \text { (100), } 179.0343(41.61), \\
173.0454(14.8), 161.0239(13.0), 135.0440(52.3), \\
134.0363(21.5), 93.0332(14.2), 85.0281(7.4)\end{array}$ & 7.00 & 529.1351 & 0.436 & $\begin{array}{l}\text { 3-caffeoyl-5-feruloylquinic } \\
\text { acid } 1,2,3,4\end{array}$ & [28] \\
\hline 19 & 529.1352 & $\mathrm{C}_{26} \mathrm{H}_{25} \mathrm{O}_{12}$ & $\begin{array}{c}529.1352 \text { (66.9), } 367.1044(100), 193.0504(12.1), \\
179.0333(57.2), 173.0447(76.9), 161.0236(15.6), \\
135.0439(73.7), 134.0365(49.6), 93.0331(76.7)\end{array}$ & 7.12 & 529.1351 & 0.077 & $\begin{array}{l}\text { 4-feruloyl-5-caffeoylquinic } \\
\text { acid } 4,5,6\end{array}$ & [28] \\
\hline 20 & 529.1361 & $\mathrm{C}_{26} \mathrm{H}_{25} \mathrm{O}_{12}$ & $\begin{array}{c}529.1361(14.2), 367.1036(60.3), 193.0499(14.5) \\
173.0447(100), 161.0239(1.1), 134.0362(17.5), 127.0392 \\
(1.0), 111.0436(3.4), 93.0330(24.9),\end{array}$ & 7.23 & 529.1351 & 1.816 & $\begin{array}{l}\text { 3-caffeoyl-4-feruloylquinic } \\
\text { acid } 1,2,3,6\end{array}$ & [28] \\
\hline
\end{tabular}


Table 2. Cont.

\begin{tabular}{|c|c|c|c|c|c|c|c|c|}
\hline Peak № & $\begin{array}{c}\text { Accurate Mass } \\
{[\mathbf{M}-\mathbf{H}]^{-} m / z}\end{array}$ & $\begin{array}{l}\text { Molecular } \\
\text { Formula }\end{array}$ & MS/MS Data $m / z$ & $t_{R} \min$ & $\begin{array}{c}\text { Exact Mass } \\
{[\mathrm{M}-\mathrm{H}]^{-} \mathrm{m} / \mathrm{z}}\end{array}$ & Delta ppm & Tentative Structure & Ref. \\
\hline 21 & 529.1388 & $\mathrm{C}_{26} \mathrm{H}_{25} \mathrm{O}_{12}$ & $\begin{array}{c}529.1388 \text { (83.7), } 353.0898(56.5), 191.0550(75.9), \\
179.0346 \text { (79.9), } 173.0448(100), 161.0237(11.9), \\
135.0439(69.8), 93.0330(38.2)\end{array}$ & 7.28 & 529.1351 & 6.880 & $\begin{array}{l}\text { 4-caffeoyl-5-feruloylquinic } \\
\text { acid } 4,5,6\end{array}$ & [28] \\
\hline 22 & 529.1356 & $\mathrm{C}_{26} \mathrm{H}_{25} \mathrm{O}_{12}$ & $\begin{array}{c}529.1356 \text { (74.2), } 367.1031(21.5), 349.0925(2.1), \\
191.0554(1.2), 179.0341(30.5), 173.0446(4.94), \\
161.0234(100), 135.0439(49.6), 93.0331(1.9)\end{array}$ & 7.34 & 529.1351 & 0.776 & $\begin{array}{l}\text { 1-feruloyl-3-caffeoylquinic } \\
\text { acid }^{1,2,3,5}\end{array}$ & [28] \\
\hline 23 & 529.1348 & $\mathrm{C}_{26} \mathrm{H}_{25} \mathrm{O}_{12}$ & $\begin{array}{c}529.1348(100), 367.1052(45.3), 179.0348(10.3), \\
173.0449(60.2), 161.0237(24.0), 135.0441(16.4), \\
111.0439(13.0), 93.0331(77.1)\end{array}$ & 7.44 & 529.1351 & -0.717 & $\begin{array}{l}\text { 4-feruloyl-?-caffeoylquinic } \\
\text { acid }\end{array}$ & $* *$ \\
\hline 24 & 529.1353 & $\mathrm{C}_{26} \mathrm{H}_{25} \mathrm{O}_{12}$ & $\begin{array}{c}529.1353(74.9), 367.1037(19.5), 349.0932(4.4), 179.0341 \\
(73.22), 161.0234(62.3), 135.0439(100), 134.0364(12.4), \\
93.0331(0.6), 85.0276(0.5)\end{array}$ & 7.71 & 529.1351 & 0.304 & $\begin{array}{l}\text { 1-feruloyl-3-caffeoylquinic } \\
\text { acid-isomer } 1,2,5,6\end{array}$ & $* *$ \\
\hline \multicolumn{9}{|c|}{ Triacylquinic acids } \\
\hline 25 & 677.1523 & $\mathrm{C}_{34} \mathrm{H}_{29} \mathrm{O}_{15}$ & $\begin{array}{c}\text { 677.1523 (2.9), } 515.1105(44.5), 353.0903(3.7), 341.0888 \\
(4.4), 191.0552(9.6), 179.0341(100), 173.0446(6.0) \\
161.0230(4.6), 135.0439 \text { (79.8), } 111.0443(1.0), 93.0331 \\
(1.6)\end{array}$ & 4.85 & 677.1512 & 1.634 & $\underset{1,2,4,5,6}{1,3,5 \text {-tricaffeoylquinic acid }}$ & [28] \\
\hline 26 & 677.1524 & $\mathrm{C}_{34} \mathrm{H}_{29} \mathrm{O}_{15}$ & $\begin{array}{c}\text { 677.1524 (89.2), } 515.1190(17.3), 353.0879(25.83), \\
341.0876(8.0), 335.0772(23.0), 323.0791(5.1), 203.1523 \\
(0.9), 191.0552(30.6), 179.0341(71.0), 173.0446(54.7), \\
161.0233(46.0), 135.0439(100), 127.0388(4.3), 111.0437 \\
(7.8), 93.0330(19.3), 85.0280(1.9)\end{array}$ & 5.26 & 677.1512 & 1.812 & $\begin{array}{l}\text { 1,3,4-tricaffeoylquinic acid } \\
1,2,3,5,6\end{array}$ & [28] \\
\hline 27 & 677.1529 & $\mathrm{C}_{34} \mathrm{H}_{29} \mathrm{O}_{15}$ & $\begin{array}{c}677.1529(52.3), 515.1200(55.7), 353.0878(26.0), \\
341.0900(15.6), 191.0553(57.3), 179.0338(75.3), \\
173.0449(31.1), 161.0236 \text { (34.66), } 135.0439(100), \\
93.0328(7.5)\end{array}$ & 5.37 & 677.1512 & 2.447 & $\begin{array}{l}\text { 1,3,5-tricaffeoylquinic acid } \\
2,5,6 \text { isomer }\end{array}$ & $* *$ \\
\hline 28 & 677.1524 & $\mathrm{C}_{34} \mathrm{H}_{29} \mathrm{O}_{15}$ & $\begin{array}{c}677.1524(87.1), 515.1281(24.3), 353.0878(26.2), \\
341.0880(31.5), 335.0787(2.3), 323.0779(10.6), \\
191.0555(34.4), 179.0342(94.0), 173.0447(78.7) \\
161.0235(21.9), 135.0439(100), 111.0439(2.2), 93.0331 \\
(24.8), 85.0279(4.8)\end{array}$ & 5.66 & 677.1512 & 1.812 & $\begin{array}{l}\text { 1,4,5-tricaffeoylquinic acid } \\
\qquad, 2,5,6\end{array}$ & [28] \\
\hline 29 & 677.1519 & $\mathrm{C}_{34} \mathrm{H}_{29} \mathrm{O}_{15}$ & $\begin{array}{c}677.1519(88.4), 515.1203(30.3), 353.0881(55.6), \\
335.0779(19.1), 203.0344(1.3), 191.0554(57.4), \\
179.0341(79.9), 173.0447(100), 161.0234(33.1) \\
135.0439(95.5), 111.0437(1.1), 93.0331(28.3), 85.0278 \\
(6.3)\end{array}$ & 7.85 & 677.1512 & 0.985 & $\begin{array}{l}\text { 3,4,5-tricaffeoylquinic acid } \\
\qquad, 2,3,4,5\end{array}$ & \\
\hline
\end{tabular}


Table 2. Cont

\begin{tabular}{|c|c|c|c|c|c|c|c|c|}
\hline Peak № & $\begin{array}{l}\text { Accurate Mass } \\
{[\mathbf{M}-\mathbf{H}]^{-} m / z}\end{array}$ & $\begin{array}{c}\text { Molecular } \\
\text { Formula }\end{array}$ & MS/MS Data $m / z$ & $t_{R} \min$ & $\begin{array}{c}\text { Exact Mass } \\
{[\mathbf{M}-\mathbf{H}]^{-} m / z}\end{array}$ & Delta ppm & Tentative Structure & Ref. \\
\hline \multicolumn{9}{|c|}{ Flavonoids } \\
\hline 30 & 269.0457 & $\mathrm{C}_{15} \mathrm{H}_{9} \mathrm{O}_{5}$ & $\begin{array}{c}269.0457(100), 151.0027(6.39), 149.0233(5.74), \\
117.0332(22.24), 107.0124(5.35)\end{array}$ & 8.73 & 269.0444 & 0.644 & apigenin $1,2,3,4,5,6$ & $*$ \\
\hline 31 & 285.0406 & $\mathrm{C}_{15} \mathrm{H}_{9} \mathrm{O}_{6}$ & $\begin{array}{c}285.0406 \text { (100), } 151.0028 \text { (5.92), } 133.0282 \text { (25.08), } \\
107.0126 \text { (3.32) }\end{array}$ & 7.82 & 285.0393 & 0.452 & luteolin $1,2,3,4,5,6$ & $*$ \\
\hline 32 & 287.0566 & $\mathrm{C}_{15} \mathrm{H}_{11} \mathrm{O}_{6}$ & $\begin{array}{c}287.0566 \text { (14.91), } 151.0025 \text { (100), } 135.0435 \text { (89.99), } \\
125.0231 \text { (5.03), } 107.0124(13.58)\end{array}$ & 7.53 & 287.0550 & 1.842 & eriodictyol $1,2,3,4,5,6$ & [24] \\
\hline 33 & 299.0561 & $\mathrm{C}_{16} \mathrm{H}_{11} \mathrm{O}_{6}$ & $\begin{array}{c}299.0561(58.00), 284.0328(100), 256.0382(0.86), \\
227.0350(3.45), 211.0393(2.22)\end{array}$ & 8.91 & 299.0550 & 0.029 & diosmetin $1,2,3,4,5,6$ & * \\
\hline 34 & 299.0562 & $\mathrm{C}_{16} \mathrm{H}_{11} \mathrm{O}_{6}$ & $\begin{array}{c}299.0562 \text { (95.99), } 284.9335(100), 227.047(2.78), \\
151.0033(3.67), 107.0123(2.84)\end{array}$ & 9.09 & 299.0550 & 0.430 & $\begin{array}{l}\text { 3,4',7-trihydroxy-3'- } \\
\text { methoxyflavone } \mathrm{e}^{1,2,3,4,5,6}\end{array}$ & [23] \\
\hline 35 & 301.0352 & $\mathrm{C}_{15} \mathrm{H}_{9} \mathrm{O}_{7}$ & $\begin{array}{c}301.0352(100), 300.0273(24.24), 178.9976(21.40), \\
151.0025(49.72), 121,0282(16.11), 107.0124(14.15)\end{array}$ & 7.83 & 301.0342 & -0.717 & quercetin $1,2,3,4,5,6$ & * \\
\hline 36 & 315.0512 & $\mathrm{C}_{16} \mathrm{H}_{11} \mathrm{O}_{7}$ & $\begin{array}{c}315.0512(61.55), 300.0279(100), 271.0252(28.24), \\
255.030(11.23), 227.0349(2.28), 136.9872(2.51)\end{array}$ & 8.34 & 315.0499 & 0.584 & nepetin ${ }^{4,5,6}$ & Mass bank \\
\hline 37 & 315.0513 & $\mathrm{C}_{16} \mathrm{H}_{11} \mathrm{O}_{7}$ & $\begin{array}{c}\text { 315.0513 (86.15), } 301.0315 \text { (11.76), } 300.0276 \text { (100), } 243 . \\
0303 \text { (0.70), } 165.9890(1.63), 136.9868 \text { (9.78) }\end{array}$ & 7.90 & 315.0499 & 0.965 & rhamnetin $1,2,3,4,5,6$ & $*$ \\
\hline 38 & 315.0514 & $\mathrm{C}_{16} \mathrm{H}_{11} \mathrm{O}_{7}$ & $\begin{array}{c}315.0514 \text { (100), } 301.0316(3.73), 300.0273 \text { (41.59), } 243 . \\
0298(1.08), 151.0025(7.85), 107.0126(6.32)\end{array}$ & 9.26 & 315.0499 & 1.156 & isorhamnetin $1,2,3,4,5,6$ & * \\
\hline 39 & 329.0670 & $\mathrm{C}_{17} \mathrm{H}_{13} \mathrm{O}_{7}$ & $\begin{array}{c}329.0607(14.45), 314.0436(100), 299.0198(25.08), \\
271.0250(47.23), 133.0282(5.34), 107.2971(0.52)\end{array}$ & 9.25 & 329.0655 & 0.954 & jaceosidin $1,3,4,6$ & Mass bank \\
\hline 40 & 331.0463 & $\mathrm{C}_{16} \mathrm{H}_{11} \mathrm{O}_{8}$ & $\begin{array}{l}331.0463 \text { (100), } 316.0226 \text { (56.40), } 287.0199 \text { (15.97), } \\
271.0246 \text { (5.47), } 270.0176 \text { (4.09), } 165.9897 \text { (19.03) }\end{array}$ & 7.80 & 331.0448 & 1.086 & patuletin $1,3,4,6$ & [23] \\
\hline 41 & 345.0618 & $\mathrm{C}_{17} \mathrm{H}_{13} \mathrm{O}_{8}$ & $\begin{array}{c}345.0618 \text { (91.18), } 330.0384(100), 315.0150(50.33), \\
287.0201(15.30), 121.0280(1.86)\end{array}$ & 8.36 & 345.0604 & 0.694 & eupatuletin $4,5,6$ & $* *$ \\
\hline 42 & 345.0618 & $\mathrm{C}_{17} \mathrm{H}_{13} \mathrm{O}_{8}$ & $\begin{array}{c}345.0618(100), 330.0385(95.66), 315.0150(46.41), \\
287.0198(14.78), 121.0284(7.72)\end{array}$ & 8.40 & 345.0604 & 0.694 & spinatoside $^{1,2,3}$ & [23] \\
\hline 43 & 345.0619 & $\mathrm{C}_{17} \mathrm{H}_{13} \mathrm{O}_{8}$ & $\begin{array}{c}345.0619(100), 330.0385(42.35), 315.0145(4.01) \\
301.0388(6.46), 287.0199(40.72)\end{array}$ & 9.38 & 345.0604 & 0.694 & spinacetin $1,2,3$ & [23] \\
\hline 44 & 359.0775 & $\mathrm{C}_{18} \mathrm{H}_{15} \mathrm{O}_{8}$ & $\begin{array}{c}359.0775 \text { (100), } 344.0539(49.89), 329.0304(52.64), \\
301.0359(6.67), 287.0139(4.46)\end{array}$ & 9.95 & 359.0761 & 0.750 & jaceidin $1,2,3,4,5,6$ & [23] \\
\hline
\end{tabular}


Table 2. Cont

\begin{tabular}{|c|c|c|c|c|c|c|c|c|}
\hline Peak № & $\begin{array}{l}\text { Accurate Mass } \\
{[\mathrm{M}-\mathrm{H}]^{-} \mathrm{m} / z}\end{array}$ & $\begin{array}{l}\text { Molecular } \\
\text { Formula }\end{array}$ & MS/MS Data $m / z$ & $t_{R} \min$ & $\begin{array}{l}\text { Exact Mass } \\
{[\mathbf{M}-\mathbf{H}]^{-} m / z}\end{array}$ & Delta ppm & Tentative Structure & Ref. \\
\hline 45 & 431.0981 & $\mathrm{C}_{21} \mathrm{H}_{19} \mathrm{O}_{10}$ & 431.0981 (100), 269.0440 (27.72), 268.0378 (57.01) & 6.17 & 431.0972 & -0.673 & $\underset{1,2,3,4,5,6}{\operatorname{apigenin}-7-\mathrm{O} \text {-glucoside }}$ & * \\
\hline 46 & 447.0934 & $\mathrm{C}_{21} \mathrm{H}_{19} \mathrm{O}_{11}$ & $\begin{array}{l}447.0934 \text { (100), } 327.0507(0.95), 285.0405(99.96), \\
151.0030(7.20), 133.0280(5.74), 107.0123(4.57)\end{array}$ & 5.45 & 447.0921 & 0.348 & $\underset{1,2,3,4,5,6}{\text { luteolin-7-O-glucoside }}$ & * \\
\hline 47 & 461.0725 & $\mathrm{C}_{21} \mathrm{H}_{17} \mathrm{O}_{12}$ & $\begin{array}{c}461.0725(42.84), 285.0406(100), 151.0025(3.72), \\
133.0280(10.72), 107.0121(1.50)\end{array}$ & 5.46 & 461.0714 & -0.150 & $\underset{1,2,3}{\text { luteolin-7-O-glucuronide }}$ & * \\
\hline 48 & 461.1094 & $\mathrm{C}_{22} \mathrm{H}_{21} \mathrm{O}_{11}$ & $\begin{array}{l}461.1094 \text { (100), } 446.0858 \text { (28.76), } 299.0554 \text { (12.48), } \\
284.0313 \text { (9.73), } 283.0250(21.41), 269.0467(2.08), \\
255.0300 \text { (73.76), } 227.0345(0.67), 151,0028(0.86)\end{array}$ & 6.37 & 461.1078 & 2.220 & diosmetin-O-glucoside ${ }^{1,2,3}$ & $* *$ \\
\hline 49 & 463.0887 & $\mathrm{C}_{21} \mathrm{H}_{19} \mathrm{O}_{12}$ & $\begin{aligned} & 463.0887(100), 372.1873(4.84), 301.0360(93.71), \\
& 300.0282(27.20)\end{aligned}$ & 4.77 & 463.0871 & 1.038 & isoquercitrin $1,2,3,4,5,6$ & * \\
\hline 50 & 463.0903 & $\mathrm{C}_{21} \mathrm{H}_{19} \mathrm{O}_{12}$ & 463.0903 (100), 301.0349 (47.50), 300.0276 (66.34) & 5.29 & 463.0871 & 4.601 & hyperoside ${ }^{1,2,3,4,5,6}$ & $*$ \\
\hline 51 & 477.1041 & $\mathrm{C}_{22} \mathrm{H}_{21} \mathrm{O}_{12}$ & $\begin{array}{c}477.1041(100), 315.0496(14.04), 314.0434(53.04) \\
300.0274(5.68), 285.0411(6.84), 243.0297(22.73) \\
271.0249(27.98), 151.0032(3.66)\end{array}$ & 6.10 & 477.1027 & 0.442 & $\begin{array}{l}\text { isorhamnetin-7-O-glucoside } \\
1,2,3,4,5,6\end{array}$ & * \\
\hline 52 & 493.0777 & $\mathrm{C}_{25} \mathrm{H}_{17} \mathrm{O}_{11}$ & $\begin{array}{c}493.0777(11.58), 315.0512(99.41), 314.0435(100), \\
300.0277(14.30), 285.0411(13.30), 243.0293(34.06), \\
227.0341(4.04), 177.0182(16.10), 151.0030(4.86), \\
133.0283(12.50)\end{array}$ & 9.12 & 493.0765 & 0.194 & caffeoyl-O-isorhamnetin ${ }^{1,3}$ & $* *$ \\
\hline 53 & 493.0974 & $\mathrm{C}_{22} \mathrm{H}_{21} \mathrm{O}_{13}$ & $\begin{array}{l}493.0974 \text { (100), } 331.0463 \text { (96.71), } 316.0224 \text { (22.61), } \\
287.0196 \text { (26.93), } 271.0253(9.58), 165.9891(8.24)\end{array}$ & 5.63 & 493.0976 & -0.257 & patuletin-O-hexoside ${ }^{4,5,6}$ & $* *$ \\
\hline 54 & 593.1504 & $\mathrm{C}_{27} \mathrm{H}_{29} \mathrm{O}_{15}$ & $\begin{array}{c}593.1504 \text { (97.39), } 318.6214(6.24), 285.0405 \text { (100), } \\
284.0327 \text { (60.74), } 227.0352(29.58)\end{array}$ & 5.71 & 593.1500 & -1.354 & $\begin{array}{l}\text { luteolin-7-O-rutinoside } \\
\qquad 1,2,3,4,5,6\end{array}$ & $*$ \\
\hline 55 & 609.1467 & $\mathrm{C}_{27} \mathrm{H}_{29} \mathrm{O}_{16}$ & $\begin{array}{c}609.1467(72.52), 343.0477(1.65), 301.0354(100) \\
300.0278(38.67), 178.9970(1.59), 151.0027 \\
(13.46), 121.0279(1.84), 107.0123(4.76)\end{array}$ & 5.17 & 609.1450 & 0.923 & rutin $1,2,3,4,5,6$ & $*$ \\
\hline 56 & 609.1472 & $\mathrm{C}_{27} \mathrm{H}_{29} \mathrm{O}_{16}$ & $\begin{array}{c}609.1472(96.84), 411.8945 \text { (5.23), } 315.0517 \text { (100), } \\
300.0275(48.87), 133.0284(7.86)\end{array}$ & 5.43 & 609.1450 & 2.149 & $\begin{array}{l}\text { isorhamnetin-O- } \\
\text { pentosyl-hexoside } \\
\text { 4,5,6 }\end{array}$ & $* *$ \\
\hline 57 & 623.1613 & $\mathrm{C}_{28} \mathrm{H}_{31} \mathrm{O}_{16}$ & $\begin{array}{c}623.1613(5.57), 315.0514(100), 301.0306(1.22) \\
300.0279 \text { (16.19), } 151.0025 \text { (7.85), } 107.0125 \text { (3.48) }\end{array}$ & 6.08 & 623.1606 & -0.703 & $\begin{array}{l}\text { isorhamnetin-3-O-rutinoside } \\
1,2,3,4,5,6\end{array}$ & $*$ \\
\hline
\end{tabular}


Table 2. Cont

\begin{tabular}{|c|c|c|c|c|c|c|c|c|}
\hline Peak № & $\begin{array}{l}\text { Accurate Mass } \\
{[\mathbf{M}-\mathbf{H}]^{-} m / z}\end{array}$ & $\begin{array}{l}\text { Molecular } \\
\text { Formula }\end{array}$ & MS/MS Data $m / z$ & $t_{R} \min$ & $\begin{array}{c}\text { Exact Mass } \\
{[\mathbf{M}-\mathbf{H}]^{-} m / z}\end{array}$ & Delta ppm & Tentative Structure & Ref. \\
\hline \multicolumn{9}{|c|}{ Sesquiterpenes } \\
\hline 58 & 229.1220 & $\mathrm{C}_{15} \mathrm{H}_{17} \mathrm{O}_{2}$ & $\begin{array}{c}229.1220 \text { (100), } 211.1116(12.69), 201.1272(19.57) \\
183.1167(40.99), 91.0548(6.40)\end{array}$ & 6.10 & 229.1223 & -3.643 & $\begin{array}{l}\text { chamazulene carboxylic } \\
\text { acid } 1,2,3\end{array}$ & [25] \\
\hline 59 & 245.1170 & $\mathrm{C}_{15} \mathrm{H}_{17} \mathrm{O}_{3}$ & $\begin{array}{c}245.1170(100), 227.1067(44.35), 217.1222(20.20) \\
201.0910(20.68), \\
\text {, } 199.1116 \text { (75.08), } 185.0959 \text { (28.15), } \\
95.0497 \text { (66.93) }\end{array}$ & 10.48 & 245.1172 & -0.942 & $\begin{array}{l}\text { dehydroleucodin/ } \\
\text { isodehydroleucodin } 1,2,3\end{array}$ & [26] \\
\hline 60 & 245.1171 & $\mathrm{C}_{15} \mathrm{H}_{17} \mathrm{O}_{3}$ & $\begin{array}{c}245.1171 \text { (84.77), } 227.1065 \text { (44.23), } 217.1225 \text { (17.55), } \\
201.0910(17.75), 199.1116(63.86), 185.0960(18.51), \\
95.0497(100)\end{array}$ & 10.48 & 245.1172 & -0.942 & $\begin{array}{l}\text { dehydroleucodin/ } \\
\text { isodehydroleucodin } 1,2,3\end{array}$ & [26] \\
\hline 61 & 247.1325 & $\mathrm{C}_{15} \mathrm{H}_{19} \mathrm{O}_{3}$ & $\begin{array}{c}247.1325(100), 229.1220(90.72), 211.1113(8.83), \\
201.1271(37.99), 187.0752(37.99), 183.1167 \text { (22.85), } \\
91.0548 \text { (9.16) }\end{array}$ & 9.79 & 247.1328 & -1.663 & $\begin{array}{c}\text { desacetoxymatricarin } \\
1,2,3,3,4,5,6\end{array}$ & $* *$ \\
\hline 62 & 249.1481 & $\mathrm{C}_{15} \mathrm{H}_{21} \mathrm{O}_{3}$ & 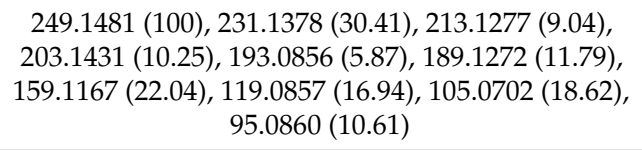 & 10.42 & 249.1485 & -0.451 & parthenolide $1,2,3,4,5,6$ & $* *$ \\
\hline 63 & 263.1275 & $\mathrm{C}_{15} \mathrm{H}_{19} \mathrm{O}_{4}$ & $\begin{array}{c}263.1275 \text { (100), } 245.1170 \text { (55.39), } 227.1061 \text { (9.88), } \\
219.1021 \text { (15.59), } 217.1223(47.56), 203.1065 \text { (10.39), } \\
199.1115(21.45), 191.0704(28.72), 95.0497 \text { (96.99) }\end{array}$ & 10.52 & 263.1277 & -0.316 & hydroxyleucodin ${ }^{1,2,3}$ & $* *$ \\
\hline 64 & 263.1274 & $\mathrm{C}_{15} \mathrm{H}_{19} \mathrm{O}_{4}$ & $\begin{array}{c}263.1274(100), 245.1171(33.08), 227.1063 \text { (4.95), } \\
219.1012(14.30), 217.1224(16.65), 203.1065 \text { (10.12), } \\
199.1117(15.19), 191.0702(79.26), 95.0497 \text { (20.99) }\end{array}$ & 11.22 & 263.1277 & -0.406 & $\begin{array}{l}\text { hydroxyleucodin isomer } \\
1,2,3\end{array}$ & $* *$ \\
\hline 65 & 263.1275 & $\mathrm{C}_{15} \mathrm{H}_{19} \mathrm{O}_{4}$ & $\begin{array}{c}263.1275(100), 245.1170(51.98), 227.1063(11.55), \\
219.1014(34.60), 217.1223(47.24), 203.1067 \text { (12.57), } \\
199.1117(22.59), 191.0702(32.46), 95.0497 \text { (81.14) }\end{array}$ & 11.22 & 263.1277 & -0.406 & $\underset{1,2,3}{\text { hydroxyleucodin isomer }}$ & $* *$ \\
\hline 66 & 265.1432 & $\mathrm{C}_{15} \mathrm{H}_{21} \mathrm{O}_{4}$ & $\begin{array}{c}265.1432(8.16), 247.1326(34.45), 229.1221(100) \\
211.1115 \text { (3.94), } 201.1273(20.45), 187.0752(14.30) \\
183.1168(7.67), 91.0548(5.66)\end{array}$ & 6.04 & 265.1434 & -0.813 & stizolin 1,3 & $* *$ \\
\hline 67 & 265.1429 & $\mathrm{C}_{15} \mathrm{H}_{21} \mathrm{O}_{4}$ & $\begin{array}{c}265.1429(24.21), 247.1325(100), 229.1222(71.63) \\
219.1372 \text { (36.25), } 201.1272(55.14), 187.1111 \text { (17.50), } \\
183.1174(17.90), 91.0548 \text { (13.40) }\end{array}$ & 9.78 & 265.1434 & -1.982 & stizolin isomer 1,3 & $* *$ \\
\hline
\end{tabular}


Table 2. Cont

\begin{tabular}{|c|c|c|c|c|c|c|c|c|}
\hline Peak № & $\begin{array}{l}\text { Accurate Mass } \\
{[\mathbf{M}-\mathbf{H}]^{-} m / z}\end{array}$ & $\begin{array}{l}\text { Molecular } \\
\text { Formula }\end{array}$ & MS/MS Data $m / z$ & $t_{R} \min$ & $\begin{array}{c}\text { Exact Mass } \\
{[\mathbf{M}-\mathbf{H}]^{-} m / z}\end{array}$ & Delta ppm & Tentative Structure & Ref. \\
\hline 68 & 305.1360 & $\mathrm{C}_{17} \mathrm{H}_{21} \mathrm{O}_{5}$ & $\begin{array}{c}305.1360(44.97), 287.1268(3.27), 269.1206(1.63), \\
263.1274(42.58), 245.1170(100), 227.1065(63.42), \\
217.1220(60.59), 109.1116(23.36), 201.0903(12.29), \\
185.0958(19.24), 181.1010(44.17), 171.1166(56.51), \\
105.0703(50.85), 95.0496(25.16)\end{array}$ & 7.05 & 305.1383 & -7.538 & matricarin $^{3}$ & $* *$ \\
\hline 69 & 305.1363 & $\mathrm{C}_{17} \mathrm{H}_{21} \mathrm{O}_{5}$ & $\begin{array}{c}305.1363(23.50), 263.1279(20.85), 245.1170(100), \\
227.1063 \text { (29.92), } 217.1216(19.51), 201.0911(2.62), \\
185.0961 \text { (16.47), } 181.1009(28.17), 171.1167(31.70), \\
131.0857(46.50), 95.0496(3.40)\end{array}$ & 5.42 & 305.1383 & -6.654 & matricarin isomer ${ }^{3}$ & $* *$ \\
\hline 70 & 307.1544 & $\mathrm{C}_{17} \mathrm{H}_{21} \mathrm{O}_{5}$ & $\begin{array}{c}307.1544(2.15), 289.1433(0.75), 267.2721(0.18), \\
247.1325(2.63), 229.1219(100), 211.1115(0.95), \\
183.1168(2.88)\end{array}$ & 9.67 & 307.1540 & 1.366 & ludalbin $1,2,3$ & $* *$ \\
\hline
\end{tabular}

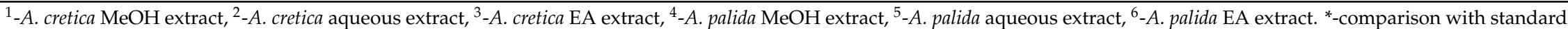
substance. ${ }^{* *}$-tentatively identification.

Table 3. Antioxidant properties of Anthemis extracts *.

\begin{tabular}{|c|c|c|c|c|c|c|c|}
\hline Plant Names & Solvents & $\begin{array}{l}\text { Phosphomolybdenum } \\
\text { (mmol TE/g) }\end{array}$ & DPPH (mg TE/g) & ABTS (mg TE/g) & $\begin{array}{l}\text { CUPRAC } \\
(\mathrm{mgTE} / \mathrm{g})\end{array}$ & FRAP (mgTE/g) & $\begin{array}{c}\text { Metal Chelating } \\
\text { Abilitiy (mg } \\
\text { EDTAE/g) }\end{array}$ \\
\hline \multirow{3}{*}{$\begin{array}{l}\text { A. tinctoria var. } \\
\text { pallida }\end{array}$} & Ethyl acetate & $2.59 \pm 0.19^{b}$ & $40.30 \pm 0.78^{\mathrm{e}}$ & $45.52 \pm 5.53^{f}$ & $113.31 \pm 2.26^{\mathrm{d}}$ & $47.63 \pm 3.77^{f}$ & $39.01 \pm 4.42^{\mathrm{a}}$ \\
\hline & Methanol & $2.99 \pm 0.14^{\mathrm{a}}$ & $407.07 \pm 8.88^{\mathrm{a}}$ & $320.11 \pm 5.67^{\mathrm{a}}$ & $691.17 \pm 12.07^{\mathrm{a}}$ & $362.12 \pm 2.63^{\mathrm{a}}$ & $28.28 \pm 1.81^{\mathrm{c}}$ \\
\hline & Aqueous & $2.65 \pm 0.02^{b}$ & $298.40 \pm 6.74^{b}$ & $303.16 \pm 8.57^{b}$ & $584.01 \pm 8.71^{\mathrm{b}}$ & $316.34 \pm 4.15^{b}$ & $33.59 \pm .16^{b}$ \\
\hline \multirow{3}{*}{$\begin{array}{l}\text { A. cretica subsp. } \\
\text { tenuiloba }\end{array}$} & Ethyl acetate & $1.69 \pm 0.08^{\mathrm{cd}}$ & $45.47 \pm 2.16^{\mathrm{e}}$ & $57.13 \pm 3.89 \mathrm{e}$ & $112.87 \pm 4.41^{\mathrm{d}}$ & $55.74 \pm 2.27^{\mathrm{e}}$ & $21.90 \pm 0.81^{\mathrm{d}}$ \\
\hline & Methanol & $1.77 \pm 0.08^{c}$ & $97.22 \pm 0.22^{c}$ & $112.41 \pm 2.35^{\mathrm{d}}$ & $223.09 \pm 6.17^{c}$ & $143.21 \pm 1.77^{\mathrm{d}}$ & $20.93 \pm 1.70 \mathrm{~d}$ \\
\hline & Aqueous & $1.56 \pm 0.05^{\mathrm{d}}$ & $86.74 \pm 2.46^{\mathrm{d}}$ & $127.68 \pm 0.45^{c}$ & $214.45 \pm 1.39^{c}$ & $130.86 \pm 1.81^{\mathrm{c}}$ & $39.64 \pm 1.34^{\mathrm{a}}$ \\
\hline
\end{tabular}

* Values expressed are means \pm S.D. of three parallel measurements. TE: Trolox equivalent; EDTAE: EDTA equivalent. Different letters indicate significant differences in the extracts $(p<0.05)$. 
Based on the experimental results (Table 3), it can be noticed that for both species, $\mathrm{MeOH}$ extract exhibited the highest total antioxidant activity. According to data presented in Table 3, ATP MeOH extract (DPPH: $407.07 \pm 8.88$ and ABTS: $320.11 \pm 5.67 \mathrm{mg} \mathrm{TE} / \mathrm{g}$ ), followed by the aqueous extract (DPPH: $298.40 \pm 6.74$ and ABTS: $303.16 \pm 8.57 \mathrm{mg}$ TE/g) showed higher radical scavenging activity in both assays. Likewise, ACT MeOH (DPPH: $97.22 \pm 0.22$ and ABTS: $112.41 \pm 2.35 \mathrm{mg}$ TE/g) and aqueous extracts (DPPH: $86.74 \pm 2.46$ and ABTS: $127.68 \pm 0.45 \mathrm{mg}$ TE/g) showed potent radical scavenging activity.

CUPRAC and FRAP assays were employed to assess the reducing capacity of different extracts. CUPRAC method evaluates the conversion of $\mathrm{Cu}$ (II) into $\mathrm{Cu}$ (I) while FRAP assay measures the reducing potential of an antioxidant reacting with the colourless TPTZ/ $\mathrm{Fe}^{3+}$ complex to form a blue $\mathrm{TPTZ} / \mathrm{Fe}^{2+}$ complex at low $\mathrm{pH}$ [30]. Remarkable reducing potencies were displayed by $\mathrm{MeOH}$ (CUPRAC: $691.17 \pm 12.07$ and FRAP: $362.12 \pm 2.63$ ) and aqueous (CUPRAC: $584.01 \pm 8.71$ and FRAP: $316.34 \pm 4.15 \mathrm{mg}$ TE/g) extracts of APT. EA extract displayed the lowest reducing capacity. This trend was also observed as regards ACT extracts (Table 3).

Chelation of pro-oxidant metals is recognized as one of the most important mechanisms of action of antioxidants. Particularly, iron is the most powerful and abundant pro-oxidant and transition metal which causes oxidative changes of cellular components, such as, lipids and proteins [31]. Evaluation of iron chelating activity showed that ATP and ACT extracts possessed notable chelation potential, with the highest activity displayed by ATP EA extract (39.01 $\pm 4.42 \mathrm{mg}$ EDTAE/g) and ACT aqueous extract $(39.64 \pm 1.34 \mathrm{mg}$ EDTAE/g).

\subsection{Enzyme Inhibitory Activity}

The enzyme inhibitory effect of ATP and ACT extracts was determined against cholinesterases, $\alpha$-amylase, $\alpha$-glucosidase, and tyrosinase and the results were presented in Table 4 . EA and $\mathrm{MeOH}$ extracts of ATP and ACT exhibited potent inhibitory activity against AChE, with the highest activity observed for ATP MeOH extract $(3.28 \pm 0.43 \mathrm{mg} \mathrm{GALAE} / \mathrm{g})$ and ACT EA extract $(4.68 \pm 0.21 \mathrm{mg}$ GALAE $/ \mathrm{g})$. On the other hand, ATP EA extract $(3.48 \pm 0.21 \mathrm{mg}$ GALAE/g), ACT EA extract $(2.51 \pm 0.34 \mathrm{mg}$ GALAE/g), and ACT MeOH extract $(1.15 \pm 0.05 \mathrm{mg}$ GALAE/g) showed inhibitory effect against $\mathrm{BChE}$. Both $\mathrm{AChE}$ and $\mathrm{BChE}$ hydrolyze acetylcholine and terminate the synaptic transmission [31]. While enhanced $\mathrm{AChE}$ activity is associated with early stages of $\mathrm{AD}, \mathrm{BChE}$ activity is found to increase with the progression of the disease. Therefore, both enzymes are considered as legitimate therapeutic targets for managing $\mathrm{AD}[32,33]$.

Table 4. Enzyme inhibitory activity of Anthemis extracts *.

\begin{tabular}{|c|c|c|c|c|c|c|}
\hline $\begin{array}{c}\text { Plant } \\
\text { Names }\end{array}$ & Solvents & $\begin{array}{c}\text { AChE } \\
\text { Inhibition } \\
\text { (mg } \\
\text { GALAE/g) }\end{array}$ & $\begin{array}{c}\text { BChE } \\
\text { Inhibition } \\
(\mathrm{mg} \\
\text { GALAE/g) }\end{array}$ & $\begin{array}{l}\text { Tyrosinase } \\
\text { Inhibition } \\
\text { (mg KAE/g) }\end{array}$ & $\begin{array}{c}\text { Amylase } \\
\text { Inhibition } \\
\text { (mmol } \\
\text { ACAE/g) }\end{array}$ & $\begin{array}{c}\text { Glucosidase } \\
\text { Inhibition } \\
\text { (mmol } \\
\text { ACAE/g) }\end{array}$ \\
\hline \multirow{3}{*}{$\begin{array}{l}\text { A. tinctoria } \\
\text { var. pallida }\end{array}$} & Ethyl acetate & $1.33 \pm 0.03^{c}$ & $3.48 \pm 0.21^{a}$ & $124.60 \pm 0.15^{b}$ & $0.78 \pm 0.05^{a}$ & $21.94 \pm 1.91^{b}$ \\
\hline & Methanol & $3.28 \pm 0.43^{b}$ & na & $124.48 \pm 1.23^{b}$ & $0.54 \pm 0.02^{c}$ & $9.15 \pm 2.26^{c}$ \\
\hline & Aqueous & na & na & $72.10 \pm 1.64^{\mathrm{d}}$ & $0.11 \pm 0.01^{\mathrm{d}}$ & $4.95 \pm 0.25^{\mathrm{d}}$ \\
\hline \multirow{3}{*}{$\begin{array}{l}\text { A. cretica } \\
\text { subsp. } \\
\text { tenuiloba }\end{array}$} & Ethyl acetate & $4.68 \pm 0.21^{a}$ & $2.51 \pm 0.34^{b}$ & $128.73 \pm 0.71^{a}$ & $0.65 \pm 0.01^{b}$ & $24.16 \pm 0.12^{a}$ \\
\hline & Methanol & $3.45 \pm 0.26^{b}$ & $1.15 \pm 0.05^{c}$ & $128.85 \pm 1.41^{\mathrm{a}}$ & $0.52 \pm 0.06^{c}$ & $4.49 \pm 0.93^{d}$ \\
\hline & Aqueous & na & na & $88.95 \pm 0.49^{c}$ & $0.09 \pm 0.01^{\mathrm{d}}$ & $2.49 \pm 0.17^{e}$ \\
\hline
\end{tabular}

${ }^{*}$ Values expressed are means \pm S.D. of three parallel measurements. GALAE: Galatamine equivalent; KAE: Kojic acid equivalent; ACAE: Acarbose equivalent; na: not active. Different letters indicate significant differences in the extracts $(p<0.05)$.

Based on the results (Table 4), it can be observed that the extracts of both species showed remarkable inhibitory activity against tyrosinase with values ranging from $124.60 \pm 0.15$ to $72.10 \pm 1.64 \mathrm{mg} \mathrm{KAE} / \mathrm{g}$ and from $128.85 \pm 1.41$ to $88.95 \pm 0.49 \mathrm{mg} \mathrm{KAE} / \mathrm{g}$ for ATP and ACT, respectively. The lowest inhibitory 
activity against tyrosinase was detected for the aqueous extracts of both species. Tyrosinase is a key enzyme in melanin biosynthesis which is responsible for skin pigmentation. However, excessive melanin production could lead to various skin disorders such as melasma, lentigines, age spots, and post-inflammatory hyperpigmentation. Thus, tyrosinase inhibitors, used as hypopigmenting agents, became increasingly important for medicinal and cosmetic products [34].

Type II diabetes is a growing pandemic and poses an enormous public health challenge for almost every country worldwide. $\alpha$-Amylase and $\alpha$-glucosidase are considered as key therapeutic targets for the management of type II diabetes. $\alpha$-Amylase and $\alpha$-glucosidase are carbohydrate hydrolysing enzymes, responsible for the breakdown of carbohydrates into glucose [35,36]. The present study showed that all ATP and ACT extracts displayed weak $\alpha$-amylase inhibitory effects, despite being active $\alpha$-glucosidase inhibitors. The highest inhibitory effect against $\alpha$-amylase $(0.78$ and 0.65 ACAEs $/ \mathrm{g}$ extract, for ATP and ACT, respectively) and $\alpha$-glucosidase (21.94 and 24.16 ACAEs/g extract, for ATP and $\mathrm{ACT}$, respectively) was displayed by EA extracts.

\subsection{Multivariate Analysis}

In an attempt to compare the difference between extract activity, multivariate statistic (sPLS-DA) was carried out by using principal component analysis (PCA) and partial least squares projection to latent structures. PCA is very useful in reducing the dimension of data with minimum loss of information. Figure 1 showed the score plots of projected extracts on principal components PC1 vs. PC2 and PC1 vs. PC3. PCA allowed analysis of biological activities disparity between the extracts. The first three components defining most of variance were found to be the best to clearly classify the extracts (Figure 1C). Nevertheless, the result obtained did not facilitate us for a better discrimination and classification of extracts. Thus, it was considered necessary to apply sPLS-DA, a supervised multivariate analysis known to offer many advantages over PCA, notably by erecting more parsimonious and easily interpretable models compared to PCA.
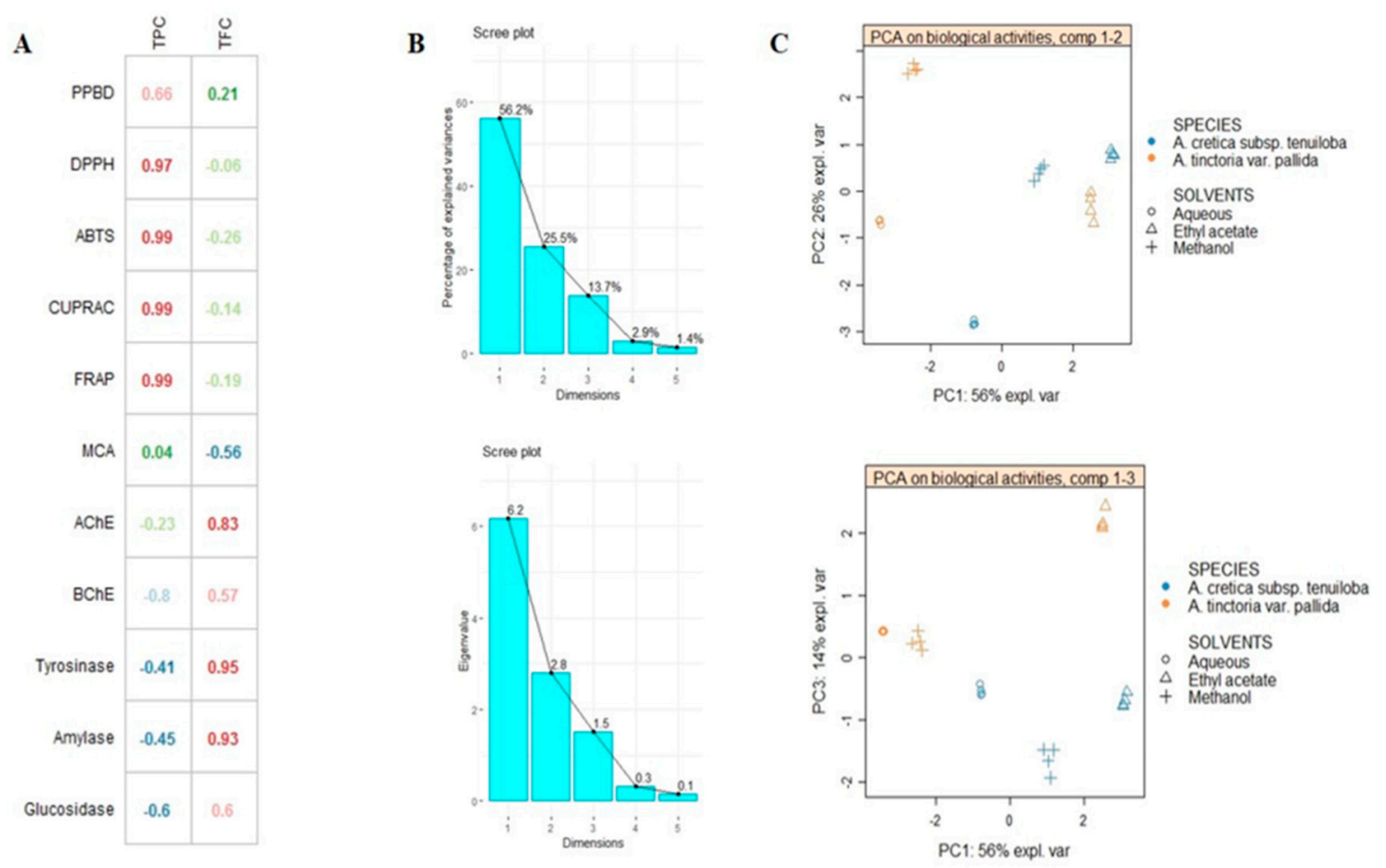

Figure 1. (A): Relationship between total phenol content (TPC), total flavonoid content (TFC) and biological activities. (B,C): results of preliminary multivariate analysis with PCA (B: Percentage of explained variance and Eigen value per component, C: PCA sample plot on PC1 vs. PC2 and PC1 vs. PC3 respectively). 
Firstly, analysed species were used as class membership criteria to assess whether they were characterized by distinctive biological activities. sPLS-DA samples plot was reported in Figure 2; as shown, a clear separation between A. cretica subsp. tenuiloba and A. tinctoria var. pallida was achieved, thus suggesting distinctive biological activities. Afterwards, with the aim to identify the most discriminant biological activities providing the differences overviewed in the sPLS-DA samples plot, VIP (variable importance in projection) plot was generated (Figure 2). Five biological activities including PPBD, DPPH, ABTS, CUPRAC and FRAP possessed a VIP score upper 1, which suggested them as discriminants for the two species.
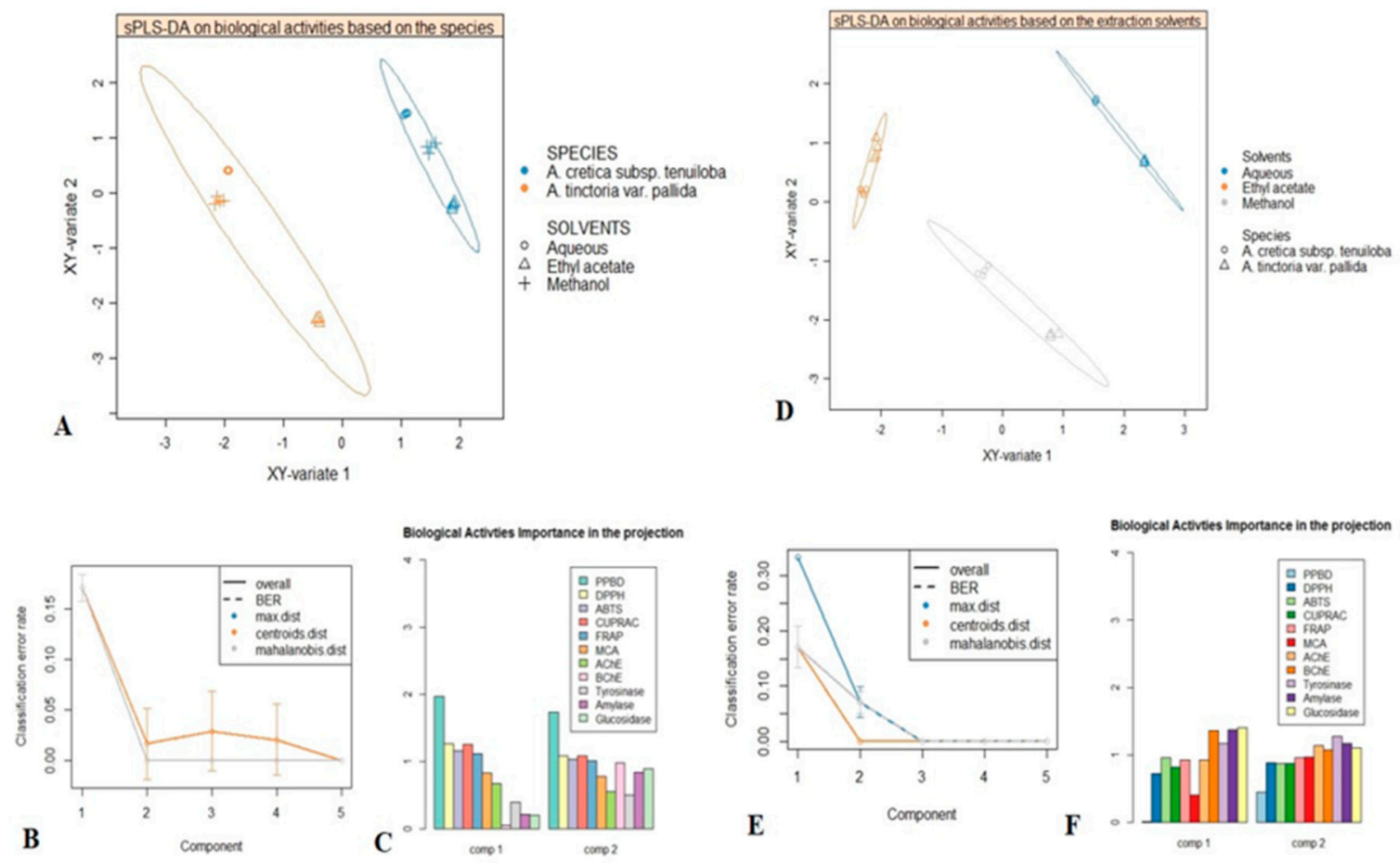

Figure 2. Supervised analysis with sPLS-DA. A: sPLS-DA samples plot with confidence ellipse plots considering the species as class membership criteria. B: Performance of the model (BER) for three prediction distances using $10 \times 5$-fold cross-validation. C: VIP score plot displaying the biological activities having highly contributed to the discrimination of both studied species. D: Factorial plan 1-2 of the sPLS-DA with confidence ellipse plots according to the extraction conditions as class membership criteria. E: The model performance per component for the three prediction distances using 5-fold cross-validation repeated 10 times. F: VIP score plot showing the biological activities outlining the difference between the three extraction conditions.

Secondly, sPLS-DA was performed considering three different extraction conditions, in order to evaluate the effect of extraction solvents on biological activities. As shown in samples plot (Figure 2) the subspace formed by the first two components showed that methanol, water and ethyl acetate extracts were well separated. Next, the prediction performance and the number of components necessary for the final model were evaluated according to BER (Balanced Error Rate). The performance of our model reached its best for two components, which suggested ncomp $=2$ for a final sPLS-DA model (Figure 2). Subsequently, the biological activities having highly contributed to the separation of used solvents were identified. As it could be seen in Figure 2, AChE, BChE, tyrosinase, $\alpha$-amylase and $\alpha$-glucosidase were the most contributing biological activities (Figure 2).

\subsection{Multidirectional Biological Evaluation}

The biological activity of Anthemis extracts was formerly evaluated through allelopathy assay, a validated pharmacognostic test for discriminating herbal extract phytotoxicity. Particularly, we 
investigated the effects of scalar extract concentrations $(100 \mu \mathrm{g} / \mathrm{mL}-10 \mathrm{mg} / \mathrm{mL})$ on seedling germination of three commercial lettuce varieties, namely Canasta (C), Romana verde (RV), and Romana bionda (RB). After challenging the seeds with Anthemis water and EA extracts, we observed that germination process was unaffected in the tested concentration range (Figure 3A-E). Conversely, ATP MeOH extract displayed concentration-dependent inhibition of seedling germination, in the range $1-10 \mathrm{mg} / \mathrm{mL}$ (Figure 3F). The root elongation rate test revealed evident inhibitory effect, in the range 1-10 mg/mL. On the other hand, extracts resulted biocompatible at the lowest tested concentration $(100 \mu \mathrm{g} / \mathrm{mL})$, with percentage elongation rate $\geq 70 \%$ compared to vehicle untreated group. The results of elongation rate test suggest a further toxicological investigation, with independent methods in order to confirm the biocompatibility limit, as described below.

A

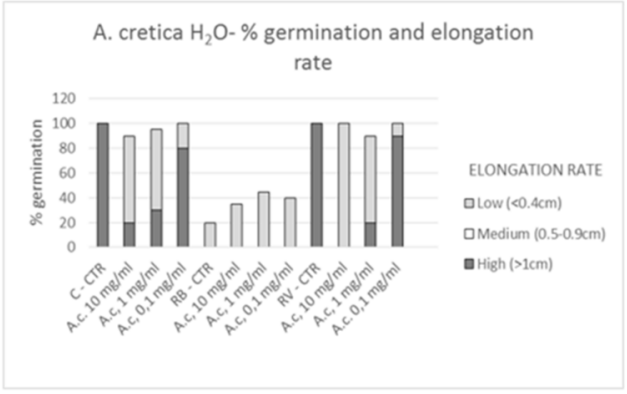

B

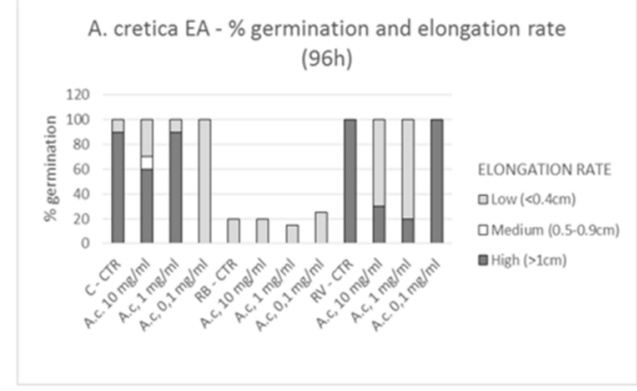

C

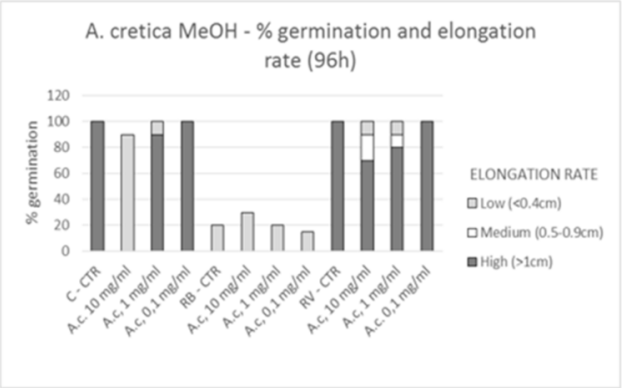

A. tinctoria $\mathrm{H}_{2} \mathrm{O}-\%$ germination and elongation rate (96h)

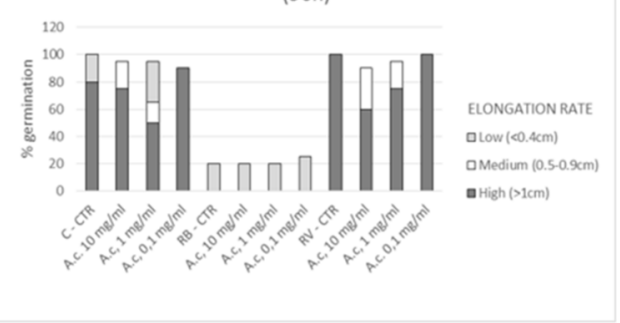

D

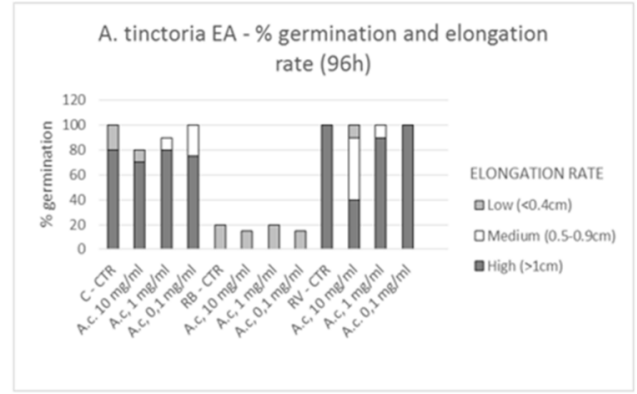

$\mathrm{E}$

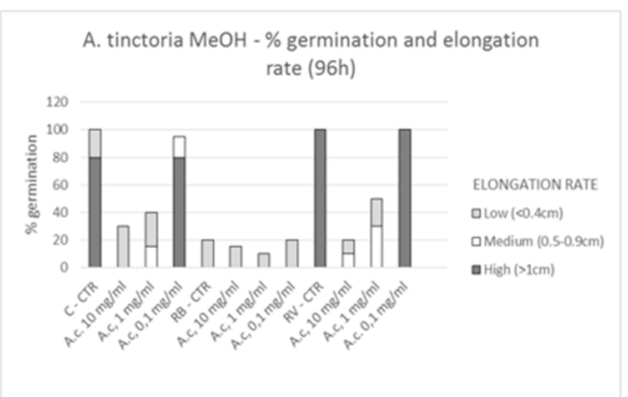

Figure 3. Seedling germination and growth of Canasta (C), Romana verde (RV) and Romana bionda (RB) seeds challenged with A. tinctoria and A. Cretica extracts. Results are expressed as root and hypocotyl (seedling) length $\pm \mathrm{SD}$ at different concentrations and mean of GP after the fourth day since the sowing. (A): Effect of A. cretica water extract on seedling germination. (B): Effect of $A$. cretica ethyl acetate (EA) extract on seedling germination. (C): Effect of $A$. cretica water methanol (MeOH) on seedling germination. (D): Effect of $A$. tinctoria water extract on seedling germination. (E): Effect of A. tinctoria ethyl acetate (EA) extract on seedling germination. (F): Effect of A. tinctoria water methanol $(\mathrm{MeOH})$ on seedling germination.

The potential toxicity of water, $\mathrm{MeOH}$ and EA extracts of Anthemis species $(0.1-20 \mathrm{mg} / \mathrm{mL})$ was also investigated through brine shrimp (Artemia salina Leach) lethality assay. Evaluation of lethality 
induced on brine shrimp, Artemia salina Leach, is considered predictive of cytotoxicity [37]. The results of this test revealed $\mathrm{LC}_{50}$ values $<10 \mathrm{mg} / \mathrm{mL}$, for all tested extracts.

Additionally, we evaluated the activity of Anthemis extracts on HypoE22 cell line viability. According to brine shrimp and allelopathy assays, we tested the extracts at $100 \mu \mathrm{g} / \mathrm{mL}$. MTT test revealed that Anthemis extracts were well tolerated by HypoE22 cells, with a resulting cell viability $\geq 70 \%$ (Figure 4). This concentration was used for subsequent ex vivo investigations aimed to elucidate extract neuroprotective effects, as following reported.

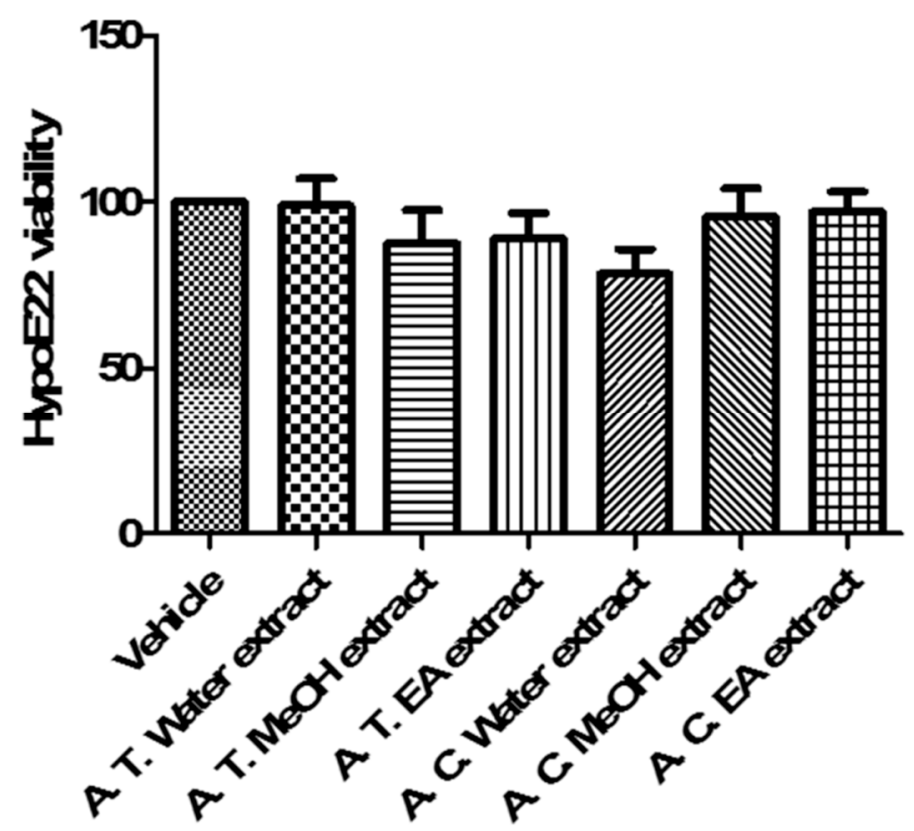

Figure 4. Effect of A. tinctoria (A. T.) and A. cretica (A. C.) extracts $(100 \mu \mathrm{g} / \mathrm{mL})$ on HypoE22 cell line viability (MTT test). Data are means \pm SD of three experiments performed in triplicate.

Cortical spreading depression (CSD) is a pathophysiological and mass depolarization of neurons and glial cells which is characterized by a change in ion and water distribution across neuron membrane associated with cytotoxic effects, including neuron death [38]. In physiological conditions, neurotransmitter release is elicited by depolarizing-stimuli $\left(\mathrm{K}^{+} 9-15 \mathrm{mM}\right)$ which, through the increased passage of $\mathrm{Ca}^{2+}$ ions across nerve terminals via voltage-sensitive calcium channels (VSCCs), stimulates classical neurotransmitter exocytosis. On the other hand, in CSD, the neurotransmitter release increases possibly through additional mechanisms, including membrane transporter reversal [39]. Particularly, excitotoxicity depolarizing-stimuli $\left(\mathrm{K}^{+} \geq 50 \mathrm{mM}\right)$ were reported to increase significantly 5-HT overflow [40] which could stimulate neurotransmitter turnover, thus explaining the cortical 5-HT depletion induced by CSD, in vivo [41]. CSD has been recently described as a potential triggering mechanism in migraine with aura, via the activation of trigeminal nociceptive system, both peripherally and centrally [42]. While low 5-HT state could play a pivotal role in migraine attack, through multiple effects, including the reduction of pain perception threshold, the increased tendency of having headache and the interference with the control of cerebrovascular nociception [41]. A chronic reduction of 5-HT is also seen in migraineurs and in depressed patients, while amitriptyline and venlafaxine are first-choice drugs for treating patients suffering from migraine with comorbid depression [43]. Considering the role played by 5-HT in anxiety and migraine [41,43], and the traditional use of Anthemis species in anxiety [18], we tested ATP and ACT extracts $(100 \mu \mathrm{g} / \mathrm{mL})$ in isolated cortex specimens challenged with an excitotoxicity stimulus constituted by $\mathrm{K}^{+}(60 \mathrm{mM})$ Krebs-Ringer buffer. The results indicated that ATP and ACT EA extracts and ACT water extracts were able to completely blunt $\mathrm{K}+(60 \mathrm{mM})$-induced 5HIIA/5-HT ratio (Figure 5), which has long been considered as a valuable index of 5-HT degradation, in the brain [44,45]. On the other hand, $\mathrm{MeOH}$ extracts were ineffective in modifying $\mathrm{K}^{+}(60 \mathrm{mM})$-induced 
5-HT degradation (Figure 5). Conversely, $\mathrm{MeOH}$ extracts revealed a more selective enzyme inhibition on AChE (Table 4). Recently, pilocarpine, a muscarinic receptor agonist, was able to antagonize CSD effects, after sub-convulsing dose administration [46], thus suggesting a role played by acetylcholine signaling stimulation, in CSD. Actually, extract capacity to improve 5-HT and acetylcholine pathways could be related to their antiradical activity (Table 3) [47]. Previously, antioxidant herbal extracts were shown to blunt oxidative stress-induced reduction of neurotransmitter level, in the brain [48,49]. Specifically, water Harpagophytum procumbens extract was able to prevent cortex 5-HT depletion induced by amyloid $\beta$-peptide [48], possibly through concomitant antioxidant mechanisms, that have been, at least partially, displayed by Anthemis extracts, as well. Whereas multiple studies also pointed out the efficacy of isolated secondary metabolites, including polyphenols and tocopherols, in blunting oxidative stress-induced monoamine depletion, thus further suggesting a putative role in managing clinical symptoms related to neurodegenerative diseases [50,51].

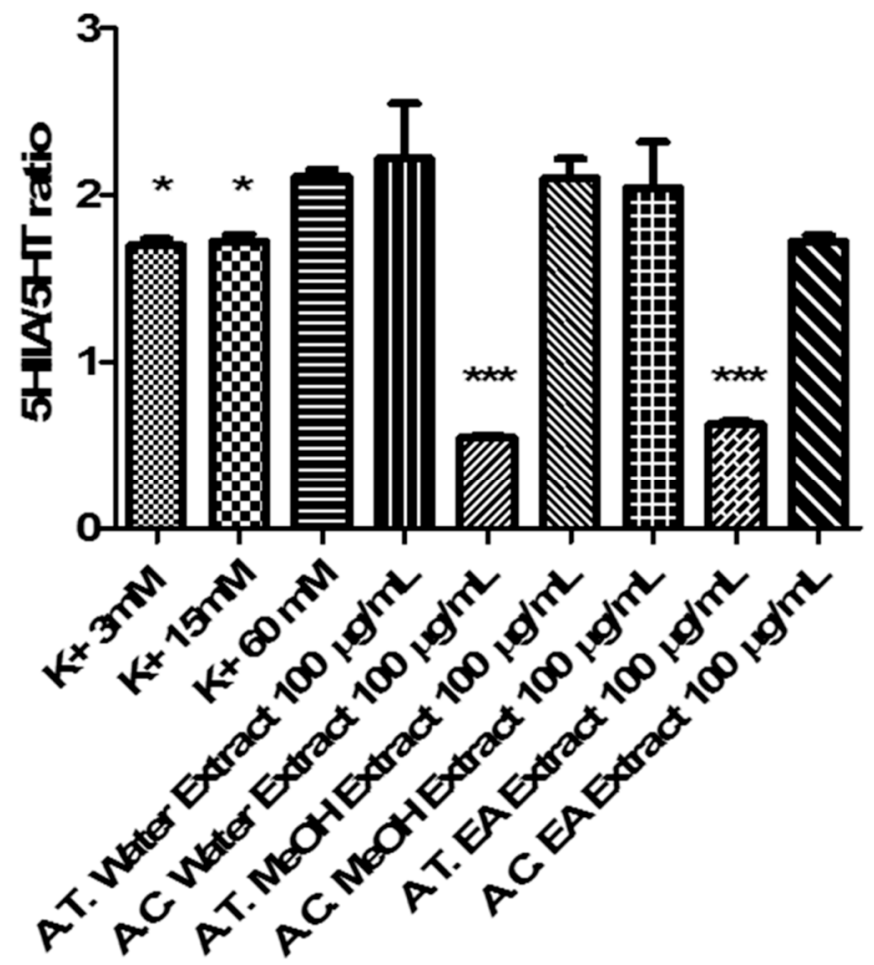

Figure 5. Effect of A. tinctoria (A. T.) and A. cretica (A. C.) extracts $(100 \mu \mathrm{g} / \mathrm{mL})$ on serotonin (5-HT) turnover, expressed as 5HIIA/5-HT ratio. Turnover was evaluated on isolated rat cortex challenged with basal $\left(\mathrm{K}^{+} 3 \mathrm{mM}\right)$ and depolarizing stimuli $\left(\mathrm{K}^{+} 15 \mathrm{mM} ; \mathrm{K}^{+} 60 \mathrm{mM}\right)$. Data are means $\pm \mathrm{SD}$ of three experiments performed in triplicate. ANOVA, $p<0.0001$; post-hoc, ${ }^{*} p<0.05$, ${ }^{* * *} p<0.001 \mathrm{vs}$. $\mathrm{K}^{+}$ $60 \mathrm{mM}$ control group.

On the other hand, after evaluating the effects of Anthemis extracts $(100 \mu \mathrm{g} / \mathrm{mL})$ on $\mathrm{LDH}$, a well-recognized marker of tissue damage [52], we observed that ACT EA and $\mathrm{MeOH}$ extracts, alongside with ATP water and EA extracts, revealed effective in blunting $\mathrm{K}^{+}(60 \mathrm{mM})$-induced LDH level (Figure 6). Considering the results of qualitative fingerprint analysis, we could hypothesize that the observed effects might be related to the presence of flavonoids and terpenes such as apigenin, patuletin, jaceosidin, quercetin, luteolin, and parthenolide. 


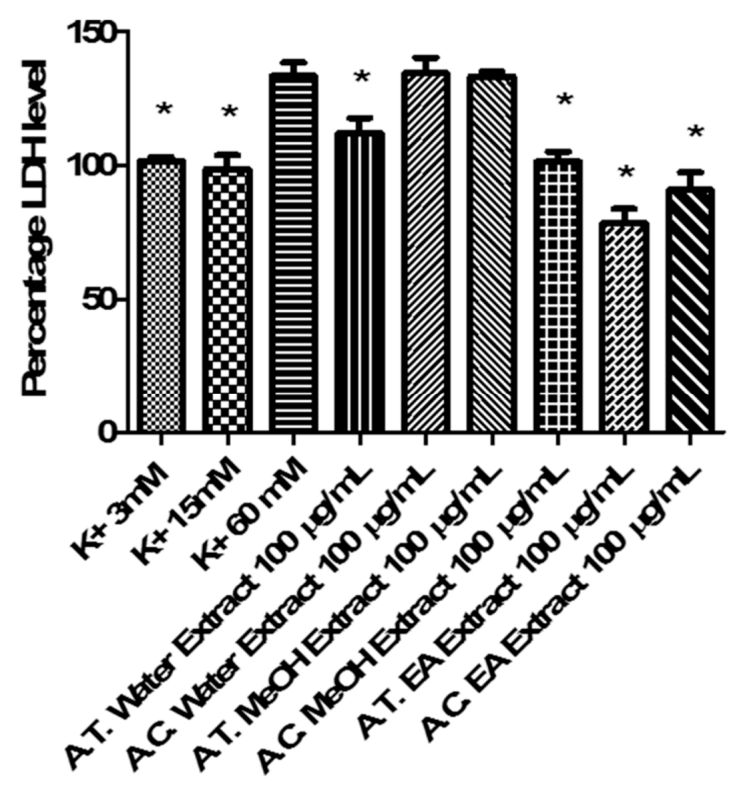

Figure 6. Effect of A. tinctoria (A. T.) and A. cretica (A. C.) extracts $(100 \mu \mathrm{g} / \mathrm{mL})$ on lactate dehydrogenase (LDH) level, measured on isolated rat cortex challenged with basal $\left(\mathrm{K}^{+} 3 \mathrm{mM}\right)$ and depolarizing stimuli $\left(\mathrm{K}^{+} 15 \mathrm{mM} ; \mathrm{K}^{+} 60 \mathrm{mM}\right)$. Data are means $\pm \mathrm{SD}$ of three experiments performed in triplicate. ANOVA, $p<0.0001$; post-hoc, ${ }^{*} p<0.05$ vs. $\mathrm{K}^{+} 60 \mathrm{mM}$ control group.

In agreement with the antiradical activity (Table 2) and blunting effect on 5-HT turnover (Figure 5), ACT water extract samples has been subjected to a further proteomic study, in order to deepen our knowledge about the putative mechanism of action related to neuroprotective effects. The deepening about ACT water extract was performed in comparison with the corresponding ATP extract that, despite showing a null effect on 5-HT turnover (Figure 5), displayed a significant inhibitory effect on $\mathrm{K}^{+}(60 \mathrm{mM})$-induced LDH level (Figure 6).

Particularly, untargeted proteomic analysis showed that $\mathrm{K}^{+} 60 \mathrm{mM}$ was able to significantly downregulate neurofilament (NFEM) proteins (Figure 7A/Supplementary Data 1), expressed along the axons and involved in axonal diameter regulation. Reduced NFEM levels have long been related to neurodegeneration [53]. The treatment of isolated rat cortex with ACT water extract was able to prevent NFEM downregulation, restoring the activity of NEFM proteins during $\mathrm{K}^{+} 15 \mathrm{mM}$ physiologic depolarizing stimulus. While ATP water extract did not exert any relevant effect on NFEM level, in isolated rat cortex challenged with $\mathrm{K}^{+} 60 \mathrm{mM}$ (Figure 7B/Supplementary Data 2). Conversely, $\mathrm{K}^{+} 60 \mathrm{mM}$ stimulus led to significant upregulation of protein $\mathrm{C}$ kinase $\gamma(\mathrm{PKC} \gamma)$ and vesicle-associated membrane protein-2 (VAMP-2) (Figure 7A/Supplementary Data 2), compared to physiologic depolarizing stimulus $\left(\mathrm{K}^{+} 15 \mathrm{mM}\right)$. VAMP-2 is placed on the membranes of neuronal endings'synaptic vesicles, playing a key role in synaptic vesicle fusion to the presynaptic neuronal ending membrane [54]. Multiple studies suggested upregulation of VAMP-2 level during hypoxia $[55,56]$, which is strictly related to high $\mathrm{K}^{+}$concentration-induced CNS injury [39]. PKC $\gamma$ plays multiple roles in neuronal cells and eye tissues, such as regulation of the neuronal receptors GRIA4/GLUR4 and GRIN1/NMDAR1, modulation of receptors and neuronal functions related to sensitivity to opiates, pain and alcohol, mediation of synaptic function and cell survival after ischemia, and inhibition of gap junction activity after oxidative stress. Its level is positively related to migraine pathogenesis [57]. Additionally, PKC $\gamma$ gene expression was observed in histidine triad nucleotide-binding protein 1 (Hint1) KO mice, that also showed increased anxiety-related behavior, compared to wild type control mice [58]. Also in this case, treatment of isolated rat cortex with ACT water extract was able to restore the activity of both VAMP-2 and PKC $\gamma$ during $\mathrm{K}^{+} 15 \mathrm{mM}$-depolarizing stimulus (Figure 7A/Supplementary Data 2), further supporting the neuroprotective effects of this extract against the burden of oxidative stress and inflammation occurring in CSD. 


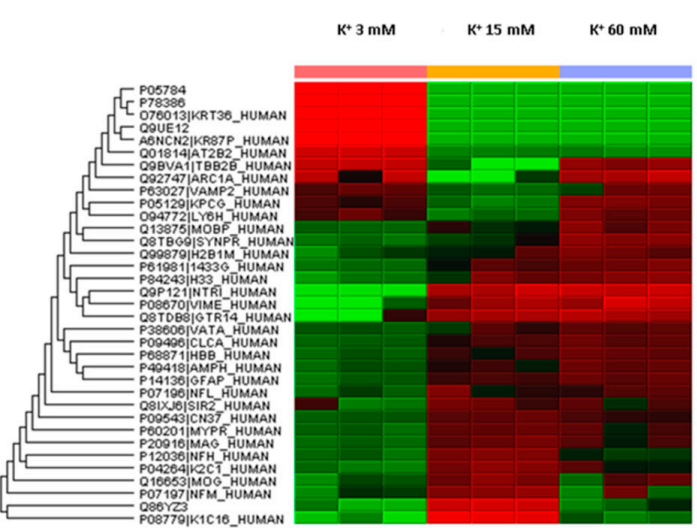

A

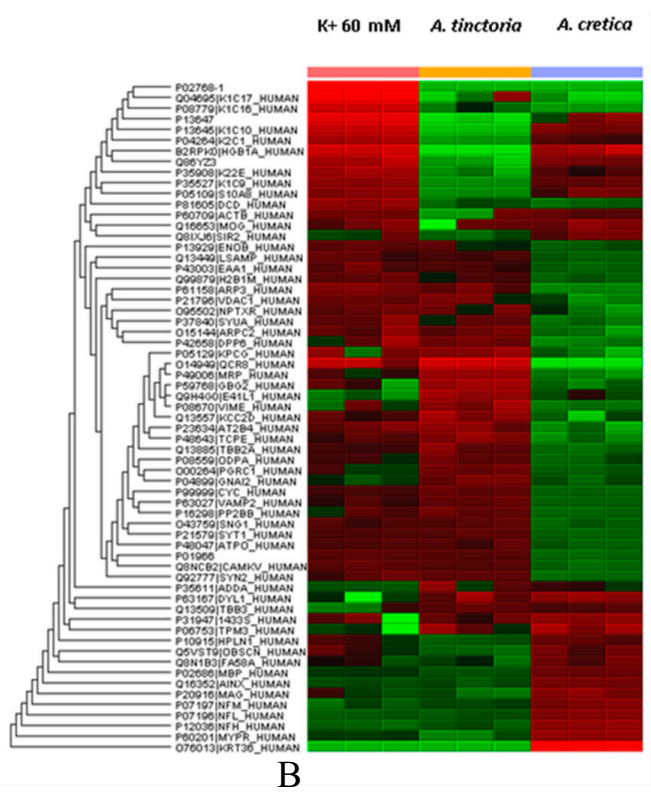

Figure 7. Panel A: Untargeted proteomic analysis performed on rat cortex challenged with basal $(\mathrm{K}+3 \mathrm{mM})$ and depolarizing stimuli $\left(\mathrm{K}^{+} 15 \mathrm{mM} ; \mathrm{K}^{+} 60 \mathrm{mM}\right)$. The activity of the detected proteins was calculated in comparison with the calibrator of the experiment $\left(\mathrm{K}^{+} 60 \mathrm{mM}\right)$. Panel B: Untargeted proteomic analysis showing the effects of $A$. tinctoria and $A$. cretica water extracts $(100 \mu \mathrm{g} / \mathrm{mL})$ on rat cortex challenged with excitotoxicity depolarizing stimulus $\left(\mathrm{K}^{+} 60 \mathrm{mM}\right)$. T The activity of the detected proteins was calculated in comparison with the calibrator of the experiment $\left(\mathrm{K}^{+} 60 \mathrm{mM}\right)$. In subfigure A, it is showed that $\mathrm{K}^{+} 60 \mathrm{mM}$ depolarizing stimulus downregulated NEFMs and upregulated VAMP-2 and PKC $\gamma$ levels. On the other hand, as depicted in subfigure B, A. cretica water extract $(100 \mu \mathrm{g} / \mathrm{mL})$ was able to restore the activity of specific proteins involved in neuron morphology and neurotransmission, including NEFMs, VAMP-2, and PKC $\gamma$. After treating rat cortex with that A. cretica water extract, the activity of these proteins was similar to that measured after challenging the brain tissue with physiologic depolarizing stimulus $\left(\mathrm{K}^{+} 15 \mathrm{mM}\right)$.

\section{Materials and Methods}

\subsection{Plant Material and Preparation of Extracts}

Sampling of the plant species (Anthemis tinctoria L. var. pallida DC. and A. cretica L. subsp. tenuiloba (DC.) Grierson) was done in Kastamonu (Hanonu) of Turkey in 2018. The collected plant identity was confirmed by the botanist Dr. Ismail Senkardes (Marmara University, Faculty of Pharmacy, Istambul, Turkey). Aerial parts were collected from wild full blooming plants and dried in ventilated oven (in the dark, temperature $40^{\circ} \mathrm{C}$ ) until constant weight. Afterwards, dried materials were powdered.

Methanol and ethyl acetate extracts were prepared through maceration method (5 $\mathrm{g}$ plant material in $100 \mathrm{~mL}$ solvents for $24 \mathrm{~h}$ ). After that, extracts were filtered and then concentrated by using one rotary evaporator under vacuo. Regarding water extracts, infusion method was selected ( $5 \mathrm{~g}$ plant was kept in $100 \mathrm{~mL}$ boiling water in $20 \mathrm{~min}$ ). The infusions were filtered and then lyophilized. All extracts were stored at $+4^{\circ} \mathrm{C}$, avoiding light exposure.

\subsection{Assays for Total Phenolic and Flavonoids}

With reference to our earlier report [59], total bioactive components namely total phenols (TPC) and flavonoids (TFC) were measured by spectrophotometric assays. Gallic acid was the standard for phenols, while rutin was selected for flavonoids. 


\subsection{Antioxidant and Enzyme Inhibition Assays}

Antioxidant properties of Anthemis extracts were determined by different in vitro assays namely FRAP, CUPRAC, DPPH, ABTS, chelating and phospomolybdenum assays. Regarding enzyme inhibitory properties, some enzymes including tyrosinase, cholinesterase, $\alpha$-amylase and $\alpha$-glucosidase were selected. All experimental procedures were given in our earlier report [59].

\subsection{UHPLC-ESI/HRMS Analysis}

Neochlorogenic acid (3-CQA) (4), chlorogenic acid (5-CQA) (6), apigenin (30), luteolin (31), quercetin (35), isoquercitrin (49), hyperoside (50), luteolin-7-O-rutinoside (54), and rutin (55) were obtained from Extrasynthese (Genay, France). 3,4-O-diCQA (12), 3,4-O-diCQA (13), diosmetin (33), rhamnetin (37), isorhamnetin (38), luteolin-7-O-glucuronide (47), isorhamnetin-7-O-glucoside (51), and isorhamnetin-3-O-rutinoside (57) were purchased from PhytoLab (Vestenbergsgreuth, Germany).

The UHPLC-ESI/HRMS analyses were carried out on a Q Exactive Plus heated electrospray ionization (HESI-II) - high resolution mass spectrometer (HRMS) (ThermoFisher Scientific, Inc., Bremen, Germany) equipped with an ultra-high-performance liquid chromatography (UHPLC) system Dionex Ultimate 3000RSLC (ThermoFisher Scientific, Inc.) [60].

\subsection{Statistical Analysis for Antioxidant and Enzyme Inhibitory Assays}

To interpret data gathered, $R$ version 3.5.1 software (The R Foundation, St. Louis, MO, USA) with corrplot and mixOmics packages was used to perform univariate and multivariate statistical analyses. One way analysis of variance (ANOVA) and Tukey's post hoc test were employed to compare bioactive compounds and biological activities between the samples. Also, relationships between bioactive compounds and biological activities were evaluated by the estimation of Pearson's correlation. For multivariate analysis, biological activities of samples were firstly analyses by PCA to pinpoint similarities or differences between samples. Then sPLS-DA was applied by using the species and different extraction condition as class memberships respectively. This allowed better comparison between the two studied species and gauged the effect of the different extraction solvents on biological activities.

\subsection{Pharmacological Assays}

\subsubsection{Allelopathy Bioassay}

Allelopathy bioassay was carried on the seeds of three commercial lettuces [Canasta (C), Romana verde (RV) and Romana bionda (RB)], because of their fast germination rate and high sensitivity. The detailed procedure has been extensively reported in our recent paper [61]. Seeds were treated with scalar Anthemis extract concentrations $(0.1-10 \mathrm{mg} / \mathrm{mL})$ and considered germinated for observed root length $\geq 1 \mathrm{~mm}$, after the third day of treatment.

\subsubsection{Artemia salina Lethality Bioassay}

Artemia salina lethality bioassay was performed as previously reported [61]. Brielfy, brine shrimp larvae were bred at $25-28^{\circ} \mathrm{C}$ for $24 \mathrm{~h}$ in presence of Anthemis extracts $(0.1-20 \mathrm{mg} / \mathrm{mL})$ dissolved in incubation medium (artificial sea water). After incubation period $(24 \mathrm{~h})$ with extracts, the number of surviving shrimps was evaluated and their vitality was compared to untreated control group. Experiments were carried out in triplicate, and percentage mortality was calculated with the following equation: $((\mathrm{T}-\mathrm{S}) / \mathrm{T}) \times 100$, where $\mathrm{T}$ and $\mathrm{S}$ are the total number of incubated larvae and survival napulii, respectively. 


\subsubsection{In Vitro Studies}

Rat hypothalamic Hypo-E22 cells were cultured in DMEM (Euroclone), as previously reported [48]. The effects of Anthemis extracts $(100 \mu \mathrm{g} / \mathrm{mL})$ on Hypo-E22 cell line viability was evaluated through 3-(4,5-dimethylthiazol-2-yl)-2,5-diphenyltetrazolium bromide (MTT) test.

\subsubsection{Ex Vivo Cortical Spreading Depression Paradigm}

Male adult Sprague-Dawley rats (200-250 g) were sacrificed by $\mathrm{CO}_{2}$ inhalation $\left(100 \% \mathrm{CO}_{2}\right.$ at a flow rate of $20 \%$ of the chamber volume per $\mathrm{min}$ ) and cortex specimens were immediately collected and maintained in thermostatic shaking bath at $37^{\circ} \mathrm{C}$ for $1 \mathrm{~h}$ (incubation period), in Krebs-Ringer buffer at different $\mathrm{K}^{+}$concentrations, as described below:

$\mathrm{K}^{+} 3 \mathrm{mM}$ : corresponding to basal condition;

$\mathrm{K}^{+} 15 \mathrm{mM}$ : corresponding to physiologic depolarizing-stimulus;

$\mathrm{K}^{+} 60 \mathrm{mM}$ : corresponding to excitotoxicity depolarizing-stimulus.

The present experimental paradigm reproduced the neural pathophysiological condition named cortical spreading depression (CSD), and was designed according to previous ex vivo and in vivo studies, describing the use of elevated $\mathrm{K}^{+}$concentrations (up to 50-60 mM) to induce central nervous system (CNS) injury [38-40]. During incubation, cortex specimens were challenged with water, $\mathrm{MeOH}$ and EA A. tinctoria and A. cretica extracts $(100 \mu \mathrm{g} / \mathrm{mL})$. Afterwards, individual cortex slices were homogenized in perchloric acid solution $(0.05 \mathrm{M})$ in order to extract and quantify serotonin $(5-\mathrm{HT})$ and its main metabolite (5-hydroxyindoleacetic acid, 5HIIA) via HPLC coupled to electrochemical detection, as previously reported [61,62]. The results were expressed as ng/mg wet tissue. Additionally, we carried out colorimetric evaluation of LDH level [52]. Finally, an untargeted proteomic profile was performed on rat cortex homogenate, as described below, in order to further elucidate the putative mechanism of action of Anthemis extracts.

\subsection{Protein Extraction and Filter-aided Sample Preparation}

After protein quantification, a volume corresponding to $50 \mathrm{ug}$ of proteins was loaded onto a Nanosep 10-kDa-cutoff filter (Pall Corporation, Michigan city, MI, USA) and digested according to the protocol we routinely use in our laboratory. Briefly, the sample was washed twice with $200 \mu \mathrm{L}$ urea buffer (8 M urea, $100 \mathrm{mM}$ Tris $\mathrm{pH} 8.5$ in milliQ water) to remove the detergents present in the lysis buffer. The proteins on the filter where subsequently reduced and alkylated by adding $100 \mu \mathrm{L}$ of DTT solution ( $8 \mathrm{mM}$ dithiothreitol in urea buffer) and $100 \mu \mathrm{L}$ of IAA solution ( $50 \mathrm{mM}$ iodoacetamide in Urea buffer). For protein digestion, the buffer was exchanged with $50 \mathrm{mM}$ ammonium bicarbonate, before adding trypsin to a ratio of 1:50 (enzyme:substrate). The reaction was incubated for $16 \mathrm{~h}$ at $37^{\circ} \mathrm{C}$, and the mixture of peptides was collected by centrifugation, acidified with $10 \%$ trifluoroacetic acid and stored at $-20{ }^{\circ} \mathrm{C}$ until analysis. The detailed description of mass spectrometric analysis is reported as "Supplementary Proteomic Analysis".

\subsection{Statistical Analysis for Pharmacological Assays}

Statistical analysis was performed using GraphPad Prism version 5.01 for Windows (GraphPad Software, San Diego, CA, USA). Means \pm S.E.M. were determined for each experimental group and analyzed by one-way analysis of variance (ANOVA), followed by Newman-Keuls comparison multiple test. Statistical significance was set at $p<0.05$. As regards the animals randomized for each experimental group, the number was calculated on the basis of the "Resource Equation" $N=(E+T) / T$ (10 $\leq \mathrm{E} \leq 20$; https://www.nc3rs.org.uk/experimental-designstatistics). 


\section{Conclusions}

Results collected in the present study indicated the promising biological effects of ATP and ACT extracts. As summarized in Figure 8, tested extracts showed significant antioxidant activity and potent inhibitory effects against key enzymes, involved in Alzheimer's disease, type II diabetes, and hyperpigmentation conditions. Particularly, EA and methanol extracts of both species showed higher enzyme inhibitory activity (at least 1.5 fold: Table 4 ) compared to water extracts. Conversely, ACT water extract revealed more significant protective effects, as evidenced by reduced $(-74 \%)$ cortex $5-\mathrm{HT}$ turnover and restored activity of key proteins (i.e., NFEMs and PKC $\gamma$ ) involved in neuron morphology and neurotransmission, in the selected model of neurotoxicity. In this context $A$. cretica water extract appears to be a good candidate for future investigations aimed to confirm and characterize the observed pharmacological effects, possibly through the use of independent experimental methods.

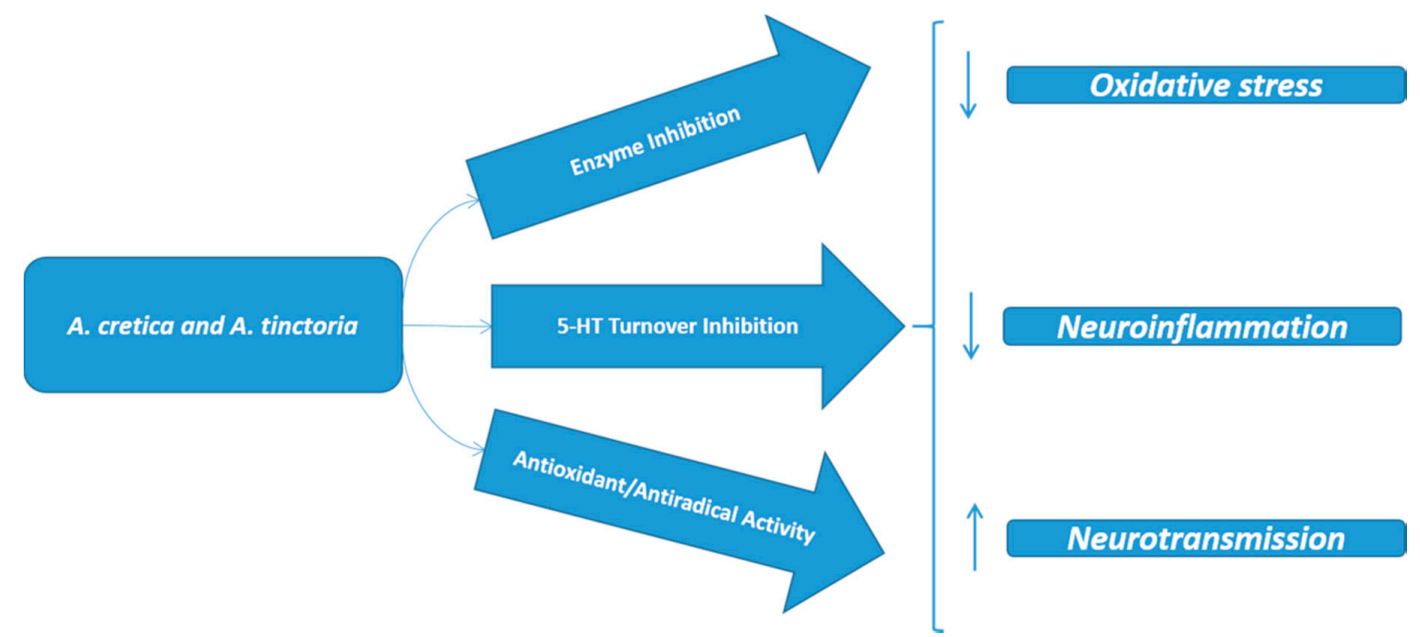

Figure 8. Protective effects induced by A. cretica and A. tinctoria extracts, as evidenced by the present pharmacological investigation.

Supplementary Materials: The following are available online. Supplementary Data 1; Supplementary Data 2; Supplementary Proteomic Analysis.

Author Contributions: Conceptualization, G.Z., L.M., G.O. and C.F.; methodology, M.R., D.Z.-D., R.G.; software, L.M.; validation, C.F., M.R., R.G. and D.Z.-D.; formal analysis, C.F., L.R.; investigation, A.C., S.L., A.M., G.M., M.P.D., S.D.S, I.S., C.M.N.P.-A., K.I.S.; resources, G.O., L.M., C.F.; data curation, C.F., G.Z.; writing-original draft preparation, C.F., G.Z.; writing-review and editing, L.M., M.F.M., G.O.; visualization, L.B.; supervision, L.B.; project administration, L.R., G.O., C.F.; funding acquisition, G.O., C.F.

Funding: This research was supported by Italian Ministry of University Grant (FAR 2017 granted to Claudio Ferrante; FAR 2017 granted to Giustino Orlando).

Conflicts of Interest: The authors declare no conflict of interest

\section{References}

1. Davis, P. Flora of Turkey and the Aegean Islands; Edinburgh University Press: Edinburgh, UK, 1975; Volume 5.

2. Doğan, G.; Demirpolat, A.; Bağcl, E. Composition of the Volatile Oils of Anthemis coelopoda var. coelopoda from Turkey. Hacettepe J. Biol. Chem. 2015, 4, 259-265. [CrossRef]

3. Staneva, J.D.; Todorova, M.N.; Evstatieva, L.N. Sesquiterpene lactones as chemotaxonomic markers in genus Anthemis. Phytochemistry 2008, 69, 607-618. [CrossRef] [PubMed]

4. Gonenc, T.; Argyropoulou, C.; Erdogan, T.; Gousiadou, C.; Juergenliemk, G.; Kıvçak, B.; Skaltsa, H. Chemical constituents from Anthemis wiedemanniana Fisch. \& Mey. Biochem. Syst. Ecol. 2011, 39, 51-55.

5. Kilic, O.; Kocak, A.; Bagci, E. Composition of the volatile oils of two Anthemis L. taxa from Turkey. Z. Für Nat. C 2011, 66, 535-540. [CrossRef] 
6. Vaverkova, S.; Habán, M.; Eerna, K. Qualitative Properties of Anthemis Tinctoria and Anthemis nobilis, (Chamaemelum nobile) under Different Environmental Conditions. Ecophysiology of Plant Production Processes in Stress Conditions. In Proceedings of the Fourth International Conference, Rackova Dolina, Slovakia, 12-14 September 2001; pp. 1-2.

7. Aboee-Mehrizi, F.; Rustaiyan, A.; Zandi, H.; Ashkezari, M.D.; Zare, M. Chemical Composition and Antimicrobial Activity of the Essential Oil of Anthemis gayana Growing in Iran. J. Essent. Oil Bear. Plants 2016, 19, 1557-1560. [CrossRef]

8. Bardaweel, S.K.; Tawaha, K.A.; Hudaib, M.M. Antioxidant, antimicrobial and antiproliferative activities of Anthemis palestina essential oil. Bmc Complementary Altern. Med. 2014, 14, 297. [CrossRef] [PubMed]

9. Kurtulmus, A.; Fafal, T.; Mert, T.; Saglam, H.; Kivcak, B.; Ozturk, T.; Demirci, B.; Baser, K. Chemical composition and antimicrobial activity of the essential oils of three Anthemis species from Turkey. Chem. Nat. Compd. 2009, 45, 900-904. [CrossRef]

10. Samadi, N.; Manayi, A.; Vazirian, M.; Samadi, M.; Zeinalzadeh, Z.; Saghari, Z.; Abadian, N.; Mozaffarian, V.-O.-A.; Khanavi, M. Chemical composition and antimicrobial activity of the essential oil of Anthemis altissima L. var. altissima. Nat. Prod. Res. 2012, 26, 1931-1934. [CrossRef] [PubMed]

11. Eser, F.; Sahin Yaglioglu, A.; Dolarslan, M.; Aktas, E.; Onal, A. Dyeing, fastness, and cytotoxic properties, and phenolic constituents of Anthemis tinctoria var. tinctoria (Asteraceae). J. Text. Inst. 2017, 108, 1489-1495. [CrossRef]

12. Kizil, S.; Kayabaşi, N.; Arslan, N. Determination of some agronomical and dyeing properties of dyer's chamomile (Anthemis Tinctoria L.). J. Cent. Eur. Agric. 2006, 6, 403-408.

13. Kültür, Ş. Medicinal plants used in Kırklareli province (Turkey). J. Ethnopharmacol. 2007, 111, 341-364. [CrossRef] [PubMed]

14. Zhang, S.; Won, Y.-K.; Ong, C.-N.; Shen, H.-M. Anti-cancer potential of sesquiterpene lactones: bioactivity and molecular mechanisms. Curr. Med. Chem. -Anti-Cancer Agents 2005, 5, 239-249. [CrossRef] [PubMed]

15. Gonenc, T.M.; Erdogan, T.F.; Demirci, B.; Baser, K.; Kivcak, B. Chemical composition of the essential oils of Anthemis coelopoda var. bourgaei and A. aciphylla var. aciphylla. Chem. Nat. Compd. 2012, 48, 332-334. [CrossRef]

16. Pavlović, M.; Kovačević, N.; Tzakou, O.; Couladis, M. Essential oil composition of Anthemis triumfetti (L.) DC. Flavour Fragr. J. 2006, 21, 297-299.

17. Uysal, I.; Celik, S.; Oldacay, M. Antimicrobial activity of Anthemis coelopoda Var. bourgaei Boiss. and Anthemis tinctoria Var. pallida DC. species having ethnobotanical features. J. Appl. Sci. 2005, 5, 639-642.

18. Özüdoğru, B.; Akaydın, G.; Erik, S.; Yesilada, E. Inferences from an ethnobotanical field expedition in the selected locations of Sivas and Yozgat provinces (Turkey). J. Ethnopharmacol. 2011, 137, 85-98. [CrossRef] [PubMed]

19. Banagozar Mohammadi, A.; Torbati, M.; Farajdokht, F.; Sadigh-Eteghad, S.; Fazljou, S.M.B.; Vatandoust, S.M.; Golzari, S.E.J.; Mahmoudi, J. Sericin alleviates restraint stress induced depressive- and anxiety-like behaviors via modulation of oxidative stress, neuroinflammation and apoptosis in the prefrontal cortex and hippocampus. Brain Res. 2019, 1715, 47-56. [CrossRef]

20. Prasanth, M.I.; Sivamaruthi, B.S.; Chaiyasut, C.; Tencomnao, T. A Review of the Role of Green Tea (Camellia sinensis) in Antiphotoaging, Stress Resistance, Neuroprotection, and Autophagy. Nutrients 2019, 11, 474. [CrossRef]

21. Clifford, M.N.; Johnston, K.L.; Knight, S.; Kuhnert, N. Hierarchical scheme for LC-MS n identification of chlorogenic acids. J. Agric. Food Chem. 2003, 51, 2900-2911. [CrossRef]

22. Clifford, M.N.; Knight, S.; Kuhnert, N. Discriminating between the six isomers of dicaffeoylquinic acid by LC-MS n. J. Agric. Food Chem. 2005, 53, 3821-3832. [CrossRef]

23. Zheleva-Dimitrova, D.; Gevrenova, R.; Zaharieva, M.M.; Najdenski, H.; Ruseva, S.; Lozanov, V.; Balabanova, V.; Yagi, S.; Momekov, G.; Mitev, V. HPLC-UV and LC-MS analyses of acylquinic acids in Geigeria alata (DC) Oliv. \& Hiern. and their contribution to antioxidant and antimicrobial capacity. Phytochem. Anal. 2017, 28, 176-184. [PubMed]

24. Justesen, U. Collision-induced fragmentation of deprotonated methoxylated flavonoids, obtained by electrospray ionization mass spectrometry. J. Mass Spectrom. 2001, 36, 169-178. [CrossRef] [PubMed]

25. Smelcerovic, A.; Lamshoeft, M.; Radulovic, N.; Ilic, D.; Palic, R. LC-MS Analysis of the Essential Oils of Achillea millefolium and Achillea crithmifolia. Chromatographia 2010, 71, 113-116. [CrossRef]

26. Priestap, H.A.; Abboud, K.A.; Velandia, A.E.; Lopez, L.A.; Barbieri, M.A. Dehydroleucodin: a guaiane-type sesquiterpene lactone. Acta Crystallogr. Sect. E Struct. Rep. Online 2011, 67, o3470. [CrossRef] [PubMed] 
27. Michalska, K.; Żylewski, M.; Kisiel, W. Structure elucidation and complete NMR spectral assignments of two new sesquiterpene lactone xylosides from Lactuca triangulata. Magn. Reson. Chem. 2008, 46, 1185-1187. [CrossRef] [PubMed]

28. Clifford, M.N.; Wu, W.; Kirkpatrick, J.; Kuhnert, N. Profiling the chlorogenic acids and other caffeic acid derivatives of herbal Chrysanthemum by LC- MS n. J. Agric. Food Chem. 2007, 55, 929-936. [CrossRef] [PubMed]

29. Lobo, V.; Patil, A.; Phatak, A.; Chandra, N. Free radicals, antioxidants and functional foods: Impact on human health. Pharmacogn. Rev. 2010, 4, 118. [CrossRef]

30. Zengin, G.; Lobine, D.; Mollica, A.; Locatelli, M.; Carradori, S.; Mahomoodally, M.F. Multiple pharmacological approaches on Fibigia eriocarpa extracts by in vitro and computational assays. Fundam. Clin. Pharmacol. 2018. [CrossRef]

31. Ma, Y.-L.; Zhu, D.-Y.; Thakur, K.; Wang, C.-H.; Wang, H.; Ren, Y.-F.; Zhang, J.-G.; Wei, Z.-J. Antioxidant and antibacterial evaluation of polysaccharides sequentially extracted from onion (Allium cepa L.). Int. J. Biol. Macromol. 2018, 111, 92-101. [CrossRef]

32. Lionetto, M.G.; Caricato, R.; Calisi, A.; Giordano, M.E.; Schettino, T. Acetylcholinesterase as a biomarker in environmental and occupational medicine: new insights and future perspectives. Biomed Res. Int. 2013, 2013. [CrossRef]

33. Greig, N.H.; Lahiri, D.K.; Sambamurti, K. Butyrylcholinesterase: an important new target in Alzheimer's disease therapy. Int. Psychogeriatr. 2002, 14, 77-91. [CrossRef] [PubMed]

34. Pintus, F.; Sabatucci, A.; Maccarrone, M.; Dainese, E.; Medda, R. Amine oxidase from Euphorbia characias: Kinetic and structural characterization. Biotechnol. Appl. Biochem. 2018, 65, 81-88. [CrossRef] [PubMed]

35. Ali, H.; Houghton, P.; Soumyanath, A. $\alpha$-Amylase inhibitory activity of some Malaysian plants used to treat diabetes; with particular reference to Phyllanthus amarus. J. Ethnopharmacol. 2006, 107, 449-455. [CrossRef] [PubMed]

36. Ouassou, H.; Zahidi, T.; Bouknana, S.; Bouhrim, M.; Mekhfi, H.; Ziyyat, A.; Aziz, M.; Bnouham, M. Inhibition of $\alpha$-Glucosidase, Intestinal Glucose Absorption, and Antidiabetic Properties by Caralluma europaea. Evid. -Based Complementary Altern. Med. 2018, 2018. [CrossRef] [PubMed]

37. Ohikhena, F.U.; Wintola, O.A.; Afolayan, A.J. Toxicity Assessment of Different Solvent Extracts of the Medicinal Plant, Phragmanthera capitata (Sprengel) Balle on Brine Shrimp (Artemia salina). Int. J. Pharmacol. 2016, 12, 701-710.

38. Richter, F.; Eitner, A.; Leuchtweis, J.; Bauer, R.; Ebersberger, A.; Lehmenkühler, A.; Schaible, H.-G. The potential of substance $P$ to initiate and perpetuate cortical spreading depression (CSD) in rat in vivo. Sci. Rep. 2018, 8, 17656. [CrossRef] [PubMed]

39. Raiteri, L.; Stigliani, S.; Zedda, L.; Raiteri, M.; Bonanno, G. Multiple mechanisms of transmitter release evoked by 'pathologically'elevated extracellular $[\mathrm{K}+]$ : involvement of transporter reversal and mitochondrial calcium. J. Neurochem. 2002, 80, 706-714. [CrossRef] [PubMed]

40. Sbrenna, S.; Marti, M.; Morari, M.; Calo, G.; Guerrini, R.; Beani, L.; Bianchi, C. Modulation of 5-hydroxytryptamine efflux from rat cortical synaptosomes by opioids and nociceptin. Br. J. Pharmacol. 2000, 130, 425-433. [CrossRef]

41. Supornsilpchai, W.; Sanguanrangsirikul, S.; Maneesri, S.; Srikiatkhachorn, A. Serotonin depletion, cortical spreading depression, and trigeminal nociception. Headache J. Head Face Pain 2006, 46, 34-39. [CrossRef]

42. Close, L.N.; Eftekhari, S.; Wang, M.; Charles, A.C.; Russo, A.F. Cortical spreading depression as a site of origin for migraine: Role of CGRP. Cephalalgia 2018, 0333102418774299. [CrossRef]

43. Zarcone, D.; Corbetta, S. Shared mechanisms of epilepsy, migraine and affective disorders. Neurol. Sci. 2018, 38, 73-76. [CrossRef] [PubMed]

44. Lee, J.; Chang, C.; Liu, I.; Chi, T.; Yu, H.; Cheng, J. Changes in endogenous monoamines in aged rats. Clin. Exp. Pharmacol. Physiol. 2001, 28, 285-289. [CrossRef] [PubMed]

45. Brunetti, L.; Orlando, G.; Ferrante, C.; Recinella, L.; Leone, S.; Chiavaroli, A.; Di Nisio, C.; Shohreh, R.; Manippa, F.; Ricciuti, A. Peripheral chemerin administration modulates hypothalamic control of feeding. Peptides 2014, 51, 115-121. [CrossRef] [PubMed]

46. Francisco, E.D.S.; Guedes, R.C. Sub-convulsing dose administration of pilocarpine reduces glycemia, increases anxiety-like behavior and decelerates cortical spreading depression in rats suckled on various litter sizes. Front. Neurosci. 2018, 12, 897. [CrossRef] [PubMed] 
47. Mollica, A.; Stefanucci, A.; Zengin, G.; Locatelli, M.; Macedonio, G.; Orlando, G.; Ferrante, C.; Menghini, L.; Recinella, L.; Leone, S. Polyphenolic composition, enzyme inhibitory effects ex-vivo and in-vivo studies on two Brassicaceae of north-central Italy. Biomed. Pharmacother. 2018, 107, 129-138. [CrossRef] [PubMed]

48. Ferrante, C.; Recinella, L.; Locatelli, M.; Guglielmi, P.; Secci, D.; Leporini, L.; Chiavaroli, A.; Leone, S.; Martinotti, S.; Brunetti, L. Protective effects induced by microwave-assisted aqueous Harpagophytum extract on rat cortex synaptosomes challenged with amyloid $\beta$-peptide. Phytother. Res. 2017, 31, 1257-1264. [CrossRef] [PubMed]

49. Romano, A.; Pace, L.; Tempesta, B.; Lavecchia, A.M.; Macheda, T.; Bedse, G.; Petrella, A.; Cifani, C.; Serviddio, G.; Vendemiale, G. Depressive-like behavior is paired to monoaminergic alteration in a murine model of Alzheimer's disease. Int. J. Neuropsychopharmacol. 2015, 18. [CrossRef]

50. Ramis, M.R.; Sarubbo, F.; Terrasa, J.L.; Moranta, D.; Aparicio, S.; Miralles, A.; Esteban, S. Chronic $\alpha$-Tocopherol Increases Central Monoamines Synthesis and Improves Cognitive and Motor Abilities in Old Rats. Rejuvenation Res. 2016, 19, 159-171. [CrossRef] [PubMed]

51. Chen, M.; Wang, T.; Yue, F.; Li, X.; Wang, P.; Li, Y.; Chan, P.; Yu, S. Tea polyphenols alleviate motor impairments, dopaminergic neuronal injury, and cerebral $\alpha$-synuclein aggregation in MPTP-intoxicated parkinsonian monkeys. Neuroscience 2015, 286, 383-392. [CrossRef] [PubMed]

52. Menghini, L.; Leporini, L.; Vecchiotti, G.; Locatelli, M.; Carradori, S.; Ferrante, C.; Zengin, G.; Recinella, L.; Chiavaroli, A.; Leone, S. Crocus sativus L. stigmas and byproducts: qualitative fingerprint, antioxidant potentials and enzyme inhibitory activities. Food Res. Int. 2018, 109, 91-98. [CrossRef]

53. Valdiglesias, V.; Fernández-Tajes, J.; Pásaro, E.; Méndez, J.; Laffon, B. Identification of differentially expressed genes in SHSY5Y cells exposed to okadaic acid by suppression subtractive hybridization. Bmc Genom. 2012, 13, 46. [CrossRef] [PubMed]

54. Jahn, R.; Südhof, T.C. Membrane fusion and exocytosis. Annu. Rev. Biochem. 1999, 68, 863-911. [CrossRef] [PubMed]

55. Manzur, A.; Sosa, M.; Seltzer, A.M. Transient increase in rab 3A and synaptobrevin immunoreactivity after mild hypoxia in neonatal rats. Cell. Mol. Neurobiol. 2001, 21, 39-52. [CrossRef] [PubMed]

56. Valdez, S.R.; Patterson, S.I.; Ezquer, M.E.; Torrecilla, M.; Lama, M.C.; Seltzer, A.M. Acute sublethal global hypoxia induces transient increase of GAP-43 immunoreactivity in the striatum of neonatal rats. Synapse 2007, 61, 124-137. [CrossRef] [PubMed]

57. Wu, B.; Wang, S.; Qin, G.; Xie, J.; Tan, G.; Zhou, J.; Chen, L. Protein kinase C $\gamma$ contributes to central sensitization in a rat model of chronic migraine. J. Mol. Neurosci. 2017, 63, 131-141. [CrossRef] [PubMed]

58. Varadarajulu, J.; Lebar, M.; Krishnamoorthy, G.; Habelt, S.; Lu, J.; Bernard Weinstein, I.; Li, H.; Holsboer, F.; Turck, C.W.; Touma, C. Increased anxiety-related behaviour in Hint1 knockout mice. Behav Brain Res. 2011, 220, 305-311. [CrossRef] [PubMed]

59. Uysal, S.; Zengin, G.; Locatelli, M.; Bahadori, M.B.; Mocan, A.; Bellagamba, G.; De Luca, E.; Mollica, A.; Aktumsek, A. Cytotoxic and enzyme inhibitory potential of two Potentilla species (P. speciosa L. and P. reptans Willd.) and their chemical composition. Front. Pharmacol. 2017, 8, 290. [CrossRef]

60. Zengin, G.; Aktumsek, A.; Ceylan, R.; Uysal, S.; Mocan, A.; Guler, G.O.; Mahomoodally, M.F.; Glamoclija, J.; Ciric, A.; Sokovic, M. Shedding light on the biological and chemical fingerprints of three Achillea species (A. biebersteinii, A. millefolium and A. teretifolia). Food Funct. 2017, 8, 1152-1165. [CrossRef]

61. Ferrante, C.; Recinella, L.; Ronci, M.; Menghini, L.; Brunetti, L.; Chiavaroli, A.; Leone, S.; Di Iorio, L.; Carradori, S.; Tirillini, B. Multiple pharmacognostic characterization on hemp commercial cultivars: Focus on inflorescence water extract activity. Food Chem. Toxicol. 2019, 125, 452-461. [CrossRef]

62. Ferrante, C.; Orlando, G.; Recinella, L.; Leone, S.; Chiavaroli, A.; Di Nisio, C.; Shohreh, R.; Manippa, F.; Ricciuti, A.; Vacca, M. Central inhibitory effects on feeding induced by the adipo-myokine irisin. Eur. J. Pharmacol. 2016, 791, 389-394. [CrossRef]

Sample Availability: Samples of the extracts are available from the authors. 